DOE/CE/23810-20B

\title{
SOLUBILITY, VISCOSITY AND DENSITY OF REFRIGERANT/LUBRICANT MIXTURES
}

Quarterly Technical Progress Report

1 April 1993 - 30 June 1993
RECEIVED

APR 251994

OSTI

David R. Henderson

\author{
Spauschus Associates, Inc. \\ 300 Corporate Center Court \\ Eagle's Landing \\ Stockbridge, GA 30281
}

October 1993

Prepared for

The Air-Conditioning and Refrigeration Technology Institute Under

ARTI MCLR Project Number 655-51200

This research project is supported, in whole or in part, by U. S. Department of Energy grant number DE-FG02-91CE23810: Materials Compatibility and Lubricants Research (MCLR) on CFC-Refrigerant Substitutes. Federal funding supporting this project constitules 93.67\% of allowable costs. Funding from non-government sources supporting this project consists of direct cost sharing of $6.33 \%$ of allowable costs; and in-kind contributions from the air-conditioning and refrigeration industry. 


\section{DISCLAIMER}

The U. S. Department of Energy's and the air-conditioning industry's support for the Materials Compatibility and Lubricants Research (MCLR) program does not constitute and endorsement by the U.S. Department of Energy, nor by the air-conditioning and refrigeration industry, of the views expressed herein.

\section{NOTICE}

This report was prepared on account of work sponsored by the United States Government. Neither the United States Government, nor the Department of Energy, nor the Air-Conditioning and Refrigeration Technology Institute, nor any of their employees, nor any of their contractors, subcontractors, or their employees, makes any warranty, expressed or implied, or assumes any iegal liability or responsibility for the accuracy, completeness, or usefulness of any information, apparatus, product or process disclosed or represents that its use would not infringe privately-owned rights.

\section{COPYRIGHT NOTICE}

(for journal publication submission)

By acceptance of this article, the publisher and/or recipient acknowledges the right of the U. S. Government and the Air-Conditioning and Refrigeration Technology Institute, Inc. (ARTI) to retain a nonexclusive, royalty-free license in and to any copyrights covering this paper.

\section{DISCLAIMER}

This report was prepared as an account of work sponsored by an agency of the United States Government. Neither the United States Government nor any agency thereof, nor any of their employees, makes any warranty, express or implied, or assumes any legal liability or responsibility for the accuracy, completeness, or usefulness of any information, apparatus, product, or process disclosed, or represents that its use would not infringe privately owned rights. Reference herein to any specific commercial product, process, or service by trade name, trademark, manufacturer, or otherwise does not necessarily constitute or imply its endorsement, recommendation, or favoring by the United States Government or any agency thereof. The views and opinions of authors expressed herein do not necessarily state or reflect those of the United States Government or any agency thereof. 


\section{TABLE OF CONTENTS}

Topic

Page

Abstract 1

Scope

Significant Results 3

CFC-12/ISO 32 Naphthenic Mineral Oil 5

CFC-12/ISO 100 Naphthenic Mineral Oil 7

HCFC-22/ISO 32 Naphthenic Mineral Oil 9

HFC-134a/ISO 22 Pentaerythritol Ester Mixed Acid 11

HFC-134a/ISO 32 Pentaerythritol Ester Mixed Acid \#1 13

HFC-134a/ISO 68 Pentaerythritol Ester Mixed Acid 15

HFC-134a/1SO 100 Pentaerythritol Ester Mixed Acid 17

HFC-134a/ISO 32 Pentaerythritol Ester Mixed Acid \#2 19

HCFC-123/ISO 32 Naphthenic Mineral Oil 21

HCFC-123/ISO 100 Naphthenic Mineral Oil 23

HCFC-123/150 SUS Alkylbenzene 25

HCFC-123/300 SUS Alkylbenzene 27

Compliance With Agreement 29

Appendix A - Corrections for Vapor Space Volume in Test Apparatus 30

Appendix B - Variations in Viscosity/Concentration Measurements Among ARTI Phase II Contractors 34

Appendix C - Lubricant Purity 39

Appendix D - Commerical Identification $\quad 40$ 


\title{
SOLUBILITY, VISCOSITY AND DENSITY \\ OF REFRIGERANT/LUBRICANT MIXTURES
}

\begin{abstract} mixtures $(0,10,20$ and 30 weight percent) of the following working fluids:

- CFC-12/ISO 32 Naphthenic Mineral Oil

- CFC-12/ISO 100 Naphthenic Mineral Oil

- HCFC-22/ISO 32 Naphthenic Mineral Oil

- HFC-134a/ISO 22 Pentaerythritol Ester Mixed Acid

- HFC-134a/ISO 32 Pentaerythritol Ester Mixed Acid \#1

- HFC-134a/ISO 68 Pentaerythritol Ester Mixed Acid

- HFC-134a/ISO 100 Pentaerythritol Ester Mixed Acid

- HFC-134a/ISO 32 Pentaerythritol Ester Mixed Acid \#2

- HCFC-123/ISO 32 Naphthenic Mineral Oil

- HCFC-123/ISO 100 Naphthenic Mineral Oil

- HCFC-123/150 SUS Alkylbenzene

- HCFC-123/300 SUS Alkylbenzene
\end{abstract}

This report presents the results of experimental measurements on low refrigerant concentration

These data have been reduced to engineering form and are presented in the form of a Daniel Chart ${ }^{1}$ and a plot of density versus temperature and composition. Extensive numerical analysis has been performed in order to derive equations which allow two independent variables (temperature and composition) and to provide for corrections in composition due to vapor space volume in the test apparatus; details of these calculations are provided in Appendix A. This report supersedes all previous reports.

\section{SCOPE}

The broad scope of this research is to measure the solubility (pressure), viscosity and density of the thirty-five refrigerant/lubricant mixtures over composition and temperature ranges as given in Table 1. Low refrigerant concentrations are generally to be measured over 0 to $100^{\circ} \mathrm{C}(273.15$ to 373.15 Kelvin), 0 to $500 \mathrm{psia}$ ( 0 to $3450 \mathrm{kPa}$ ). The experimental data are to be graphically reported in the Daniel Chart format, and mathematical relationships are to be derived.

The entire process of measurement, data reduction, mathematical modeling and plotting has been completed for the low refrigerant concentrations on fluids $1,2,3,5,6,7,8,10,13,14,15$ and 16 (Table 1) and are reported here.

'G. Daniel, M. J. Anderson, W. Schmid and M. Tokumitsu, "Performance of Selected Synthetic Lubricants in Industrial Heat Pumps," Heat Recovery Systems, Vol. 2, No. 4, 1982. pp. 359-368. 
Table 1: Refrigerant/Lubricant Mixtures Under Study

\section{Refrigerant/Lubricant}

1. $\quad 12 / \mathrm{ISO} 32 \mathrm{MO}^{3}$

2. $12 / \mathrm{ISO} 100 \mathrm{MO}$

3. $22 / 1 \mathrm{SO} 32 \mathrm{MO}$

4. $134 \mathrm{a} / \mathrm{ISO} 68 \mathrm{PAG}^{4}$

5. 134a/ISO 22 POE-MA

6. 134a/ISO 32 POE-MA\#1

7. $134 \mathrm{a} / \mathrm{ISO} 68$ POE-MA

8. 134a/ISO 100 POE-MA

9. $134 \mathrm{a} /$ ISO 22 POE-BA ${ }^{6}$

10. 134a/ISO 32 POE-MA\#2

11. 134a/ISO 68 POE-BA

12. 134a/ISO 100 POE-BA

13. 123/ISO $32 \mathrm{MO}$

14. 123/ISO $100 \mathrm{MO}$

15. $123 / 150$ SUS $\mathrm{AB}^{7}$

16. $123 / 300$ SUS AB

17. 32/ISO 22 POE-MA

18. 32/ISO 68 POE-MA

19. 32/ISO 32 POE-BA

20. 32/ISO 100 POE-BA

21. 125/ISO 22 POE-MA

22. 125/ISO 68 POE-MA

23. 125/ISO 32 POE-BA

24. 125/ISO 100 POE-BA

25. $152 \mathrm{a} / 150$ SUS $\mathrm{AB}$

26. $152 \mathrm{a} / 300$ SUS AB

27. 152a/ISO 22 POE-MA

28. 152a/ISO 68 POE-MA

29. 143a/ISO 22 POE-MA

30. 143a/ISO 68 POE-MA

31. 143a/ISO 32 POE-BA

32. 143a/ISO 100 POE-MA

33. $124 / 150$ SUS AB

34. $124 / 300$ SUS AB

35. $142 \mathrm{~b} / \mathrm{ISO} 32 \mathrm{AB}$
Low Refrigerant

Concentrations ${ }^{1}$

Temp, Range, $\mathrm{C}$

0 to 100

0 to 100

0 to 100

0 to 100

0 to 100

0 to 100

0 to 100

0 to 100

0 to 100

0 to 100

0 to 100

0 to 100

0 to 100

0 to 100

0 to 100

0 to 100

0 to 75

0 to 75

0 to 75

0 to 75

0 to 65

0 to 65

0 to 65

0 to 65

0 to 100

0 to 100

0 to 100

0 to 100

0 to 70

0 to 70

0 to 70

0 to 70

0 to 100

0 to 100

0 to 100
High Refrigerant Concentrations ${ }^{2}$ Temp, Range, C

-40 to +40

-40 to +40

-40 to +40

-40 to +40

-40 to +40

-40 to +40

-40 to +40

-40 to +40

-40 to +40

-40 to +40

-40 to +40

-40 to +40

-20 to +40

-20 to +40

-20 to +40

-20 to +40

-50 to +40

-50 to +40

-50 to +40

-50 to +40

-40 to +40

-40 to +40

-40 to +40

-40 to +40

-40 to +40

-40 to +40

-40 to +40

-40 to +40

-45 to +40

-45 to +40

-45 to +40

-45 to +40

-40 to +40

-40 to +40

-40 to +40

'Low Refrigerant Concentrations are $0,10,20$ and 30 weight percent refrigerant.

${ }^{2}$ High Refrigerant Concentrations are 80, 90 and 100 weight percent refrigerant.

${ }^{3}$ Mineral Oil

${ }^{4}$ Polyalkylene Glycol (butyl monoether)

'Polyolester (Pentaerythritol) - Mixed Acid

'Polyolester (Pentaerythritol) - Branched Acid

'Alkylbenzene 


\section{SIGNIFICANT RESULTS}

Experimental data are presented in the form of mathematical models and two charts, the first giving the density, the second giving the viscosity and solubility (pressure) as functions of temperature and composition (Daniel Chart). On the upper portion of the Daniel Chart are isobaric viscosity curves which have been algebraically generated from the measured data.

In order to generate these isobaric curves, the assumption has been made that interpolation between measured composition curves is valid over the temperature range for which data has been obtained. It is also assumed that these fluids are two component mixtures having one liquid phase and one vapor phase; application of the Gibbs Phase Rule then gives two degrees of freedom.

The charts follow the numbering system given in Table 1 and are thus not consecutively numbered in this Quarterly Report due to fluid availability and internal scheduling of experimental runs. Accompanying each set of charts is a table of regression constants and correlation coefficients which is explained below.

Functions of two independent variables are chosen to represent the experimental data; generally, the simplest form which represents the data accurately has been employed. The relationship chosen to represent the viscosity data is a modified form of the Walther equation:

$$
\begin{aligned}
& \log \{\log (\eta+0.7)\}=\left\{a_{1}+a_{2} \cdot \log (T)\right\}+\omega \cdot\left\{a_{3}+a_{4} \cdot \log (T)\right\}+\omega^{2} \cdot\left\{a_{5}+a_{6} \cdot \log (T)\right\} \\
& \text { where } \\
& \eta=\text { absolute viscosity, centipoise } \\
& \mathrm{T}=\text { Temperature, Kelvin } \\
& \omega=\text { mass fraction refrigerant } \\
& \log =\operatorname{logarithm} \text { to the base } 10 \\
& a_{1} \text { through } a_{6}=\text { constants. }
\end{aligned}
$$

Vapor pressure is given by

$$
P=\left\{a_{1}+a_{2} \cdot T+a_{3} \cdot T^{2}\right\}+\omega \cdot\left\{a_{4}+a_{9} \cdot T+a_{6} \cdot T^{2}\right\}
$$

where

$$
\begin{aligned}
& P=\text { pressure, kilopascals } \\
& T=\text { Temperature, Kelvin } \\
& \omega=\text { mass fraction refrigerant } \\
& a_{1} \text { through } a_{6}=\text { constants (different from equation } 1 \text { ). }
\end{aligned}
$$

Density is given by

$$
\rho=\left\{a_{1}+a_{2} \cdot T\right\}+\omega \cdot\left\{a_{3}+a_{4} \cdot T\right\}+\omega^{2} \cdot\left\{a_{5}+a_{6} \cdot T\right\}
$$

where

$$
\begin{aligned}
& \rho=\text { density, } g / c c \\
& T=\text { Temperature, Kelvin } \\
& \omega=\text { mass fraction refrigerant } \\
& a_{1} \text { through } a_{6}=\text { constants (different from equations } 1,2 \text { ). }
\end{aligned}
$$


Kinematic viscosity is given by

$$
\begin{array}{cl}
\log \{\log (v+0.7)\}= & \left\{a_{1}+a_{2} \cdot \log (T)\right\}+\omega \cdot\left\{a_{3}+a_{4} \log (T)\right\}+\omega^{2} \cdot\left\{a_{5}+a_{6} \log (T)\right\} \\
\text { where } & \\
& v=\text { kinematic viscosity, centistoke } \\
& T=\text { Temperature, Kelvin } \\
& \omega=\text { mass fraction refrigerant } \\
& \log =\operatorname{logarithm} \text { to the base } 10 \\
& \left.a_{1} \text { through } a_{6}=\text { constants (different from equations } 1,2,3\right) .
\end{array}
$$

Multivariate correlation coefficients are given as a measure of how well the regression equations fit the data. These coefficients, denoted by $\sigma$, are the square root of the explained variation divided by the total variation. Mathematically, we have

$$
\begin{aligned}
& \text { Unexplained variation }=\Sigma\left(y_{i}-y_{c}\right)^{2} \\
& \text { Total variation }=\Sigma\left(y_{1}-y_{\mathrm{av}}\right)^{2} \\
& \text { Explained variation }=\text { Total variation - Unexplained variation }
\end{aligned}
$$

where

$$
\begin{aligned}
& y_{1}=\text { experimental data point } \\
& y_{c}=\text { calculated data point } \\
& y_{a v}=\text { average of experimental data points }
\end{aligned}
$$

giving

$$
\sigma=\sqrt{\frac{\Sigma\left(y_{i}-y_{s v}\right)^{2}-\Sigma\left(y_{i}-y_{c}\right)^{2}}{\Sigma\left(y_{i}-y_{s v}\right)^{2}}}
$$

Results to date begin on the next page and constitute the body of this report. 
Table 1-1: Viscosi '-, Solubility and Density Parameters CFC-12/ISO 32 Naphthenic Mineral Oil

$\begin{array}{lccc}\begin{array}{l}\text { Dynamic } \\ \text { Viscosity } \\ \text { (eq. 1) }\end{array} & \begin{array}{c}\text { Vapor } \\ \text { Pressure } \\ \text { (eq. 2) }\end{array} & \begin{array}{c}\text { Denisty } \\ \text { (eq. 3) }\end{array} & \begin{array}{c}\text { Kinematic } \\ \text { Viscosity } \\ \text { (eq. 4) }\end{array} \\ & & & 1.091796 \\ 128863 \mathrm{E}+1 & -2.226863 \mathrm{E}+3 & 1.072288 \mathrm{E}+1 \\ -4.459166 & 1.386308 \mathrm{E}+1 & -6.306535 \mathrm{E}-4 & -4.226366 \\ .524742 \mathrm{E}-1 & -2.114996 \mathrm{E}-2 & 4.040375 \mathrm{E}-1 & 1.127096 \\ -5.434154 \mathrm{E}-1 & 2.802944 \mathrm{E}+4 & -2.762705 \mathrm{E}-4 & -8.203340 \mathrm{E}-1 \\ .189490 \mathrm{E}+1 & -2.141093 \mathrm{E}+2 & 6.946489 \mathrm{E}-1 & -2.014214 \mathrm{E}+1 \\ 8.513484 & 4.187154 \mathrm{E}-1 & -1.681253 \mathrm{E}-3 & 7.745700 \\ 0.9993 & 0.9970 & 0.9997 & 0.9991\end{array}$

Figure 1-1: Density of CFC-12/ISO 32 Naphthenic Mineral Oil

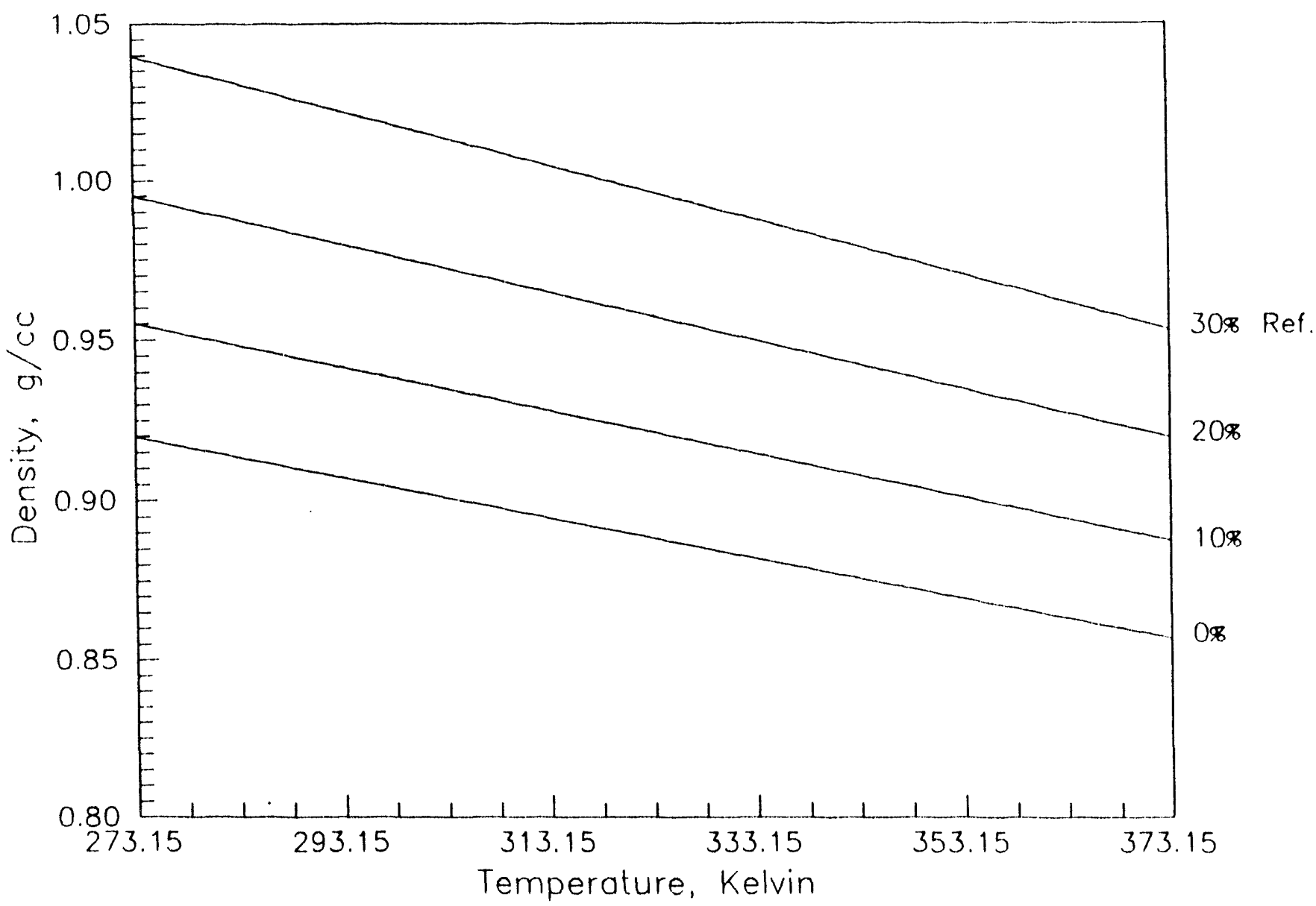


Figure 1-2: Viscosity and Solubility of CFC-12/ISO 32 Naphthenic Mineral Oil

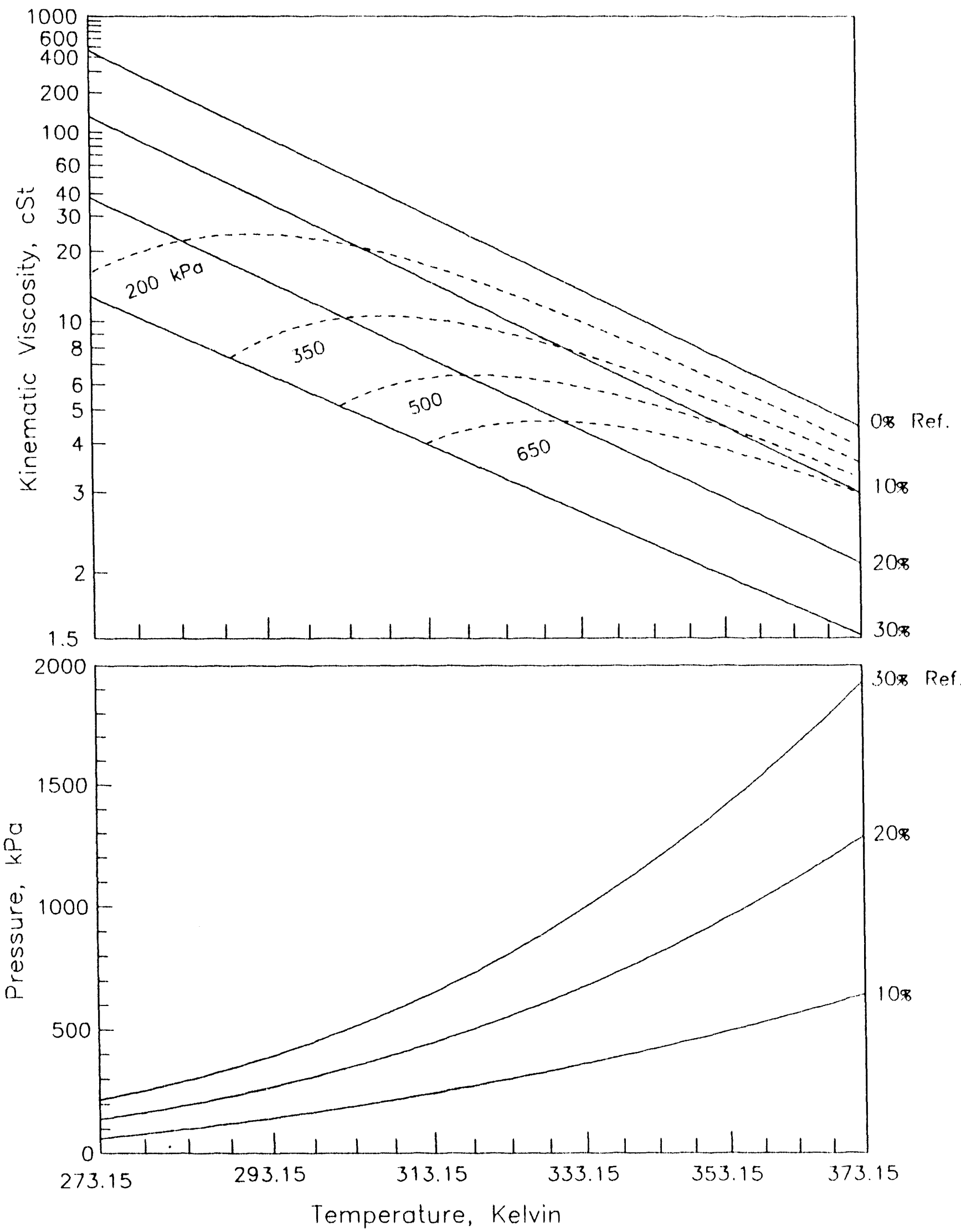


Table 2-1: Viscosity, Solubility and Density Parameters

CFC-12/ISO 100 Naphthenic Mineral Oil

$\begin{array}{cccc}\text { Dynamic } & \text { Vapor } & & \text { Kinematic } \\ \text { Viscosity } & \text { Pressure } & \text { Denisty } & \text { Viscosity } \\ \text { (eq. 1) } & \text { (eq. 2) } & \text { (eq. 3) } & \text { (eq. 4) }\end{array}$

$\begin{array}{lcccc}a_{1} & 1.104244 \mathrm{E}+1 & -3.372559 \mathrm{E}+3 & 1.094413 & 1.055208 \mathrm{E}+1 \\ a_{2} & -4.308084 & 2.082366 \mathrm{E}+1 & -6.153593 \mathrm{E}-4 & -4.107731 \\ a_{3} & -4.793902 \mathrm{E}-1 & -3.144160 \mathrm{E}-2 & 5.388011 \mathrm{E}-1 & -5.047290 \mathrm{E}-2 \\ \mathrm{a}_{4} & -2.210834 \mathrm{E}-1 & 3.271269 \mathrm{E}+4 & -7.570955 \mathrm{E}-4 & -3.874498 \mathrm{E}-1 \\ a_{5} & -6.383261 & -2.500250 \mathrm{E}+2 & 1.869725 \mathrm{E}-1 & -4.621944 \\ a_{6} & 2.392385 & 4.876217 \mathrm{E}-1 & 8.880958 \mathrm{E}-5 & 1.618327 \\ \sigma & 0.9997 & 0.9924 & 0.9997 & 0.9998\end{array}$

Figure 2-1: Density of CFC-12/ISO 100 Naphthenic Mineral Oil

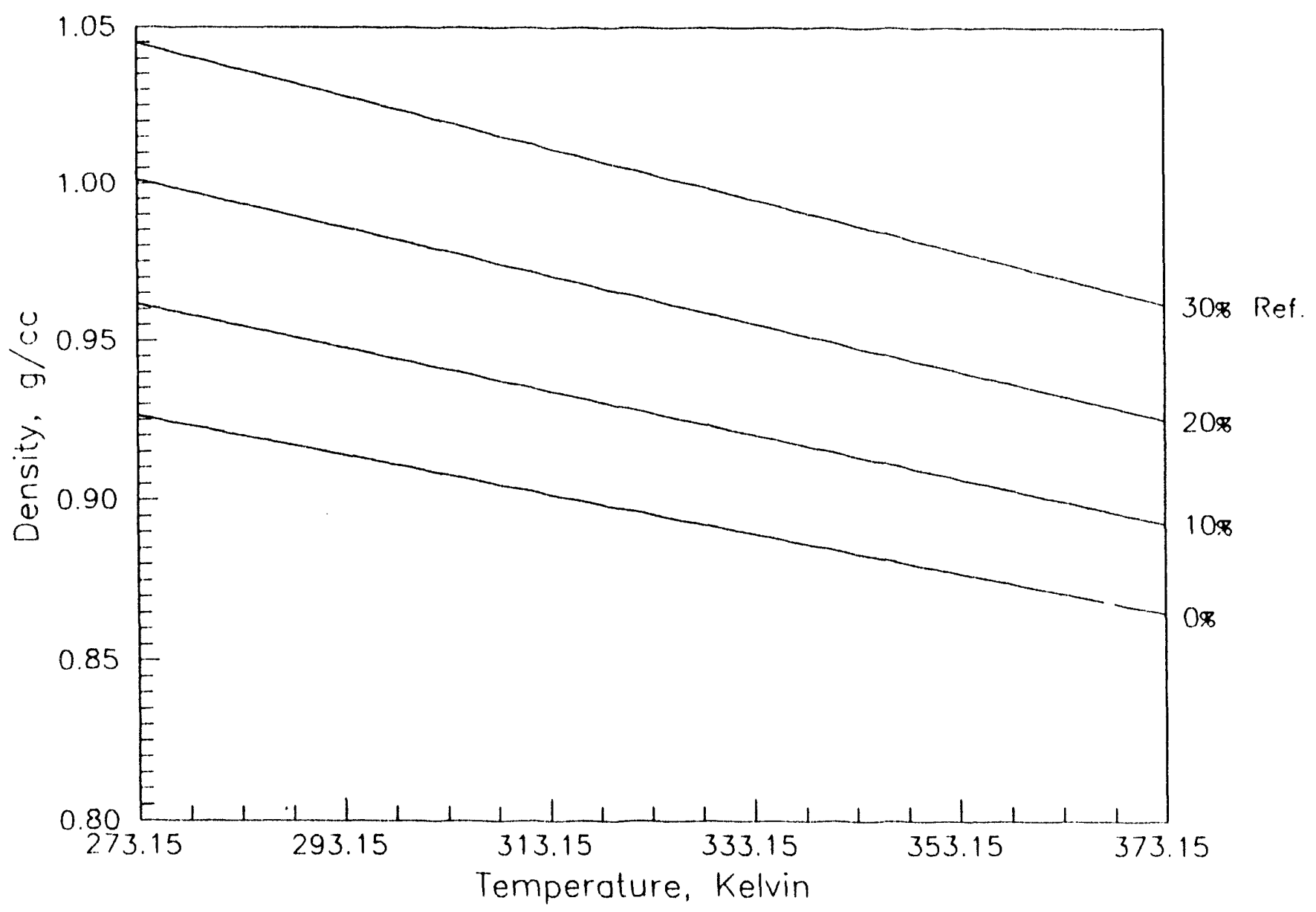


Figure 2-2: Viscosity and Solubility of CFC-12/ISO 100 Naphthenic Mineral Oil

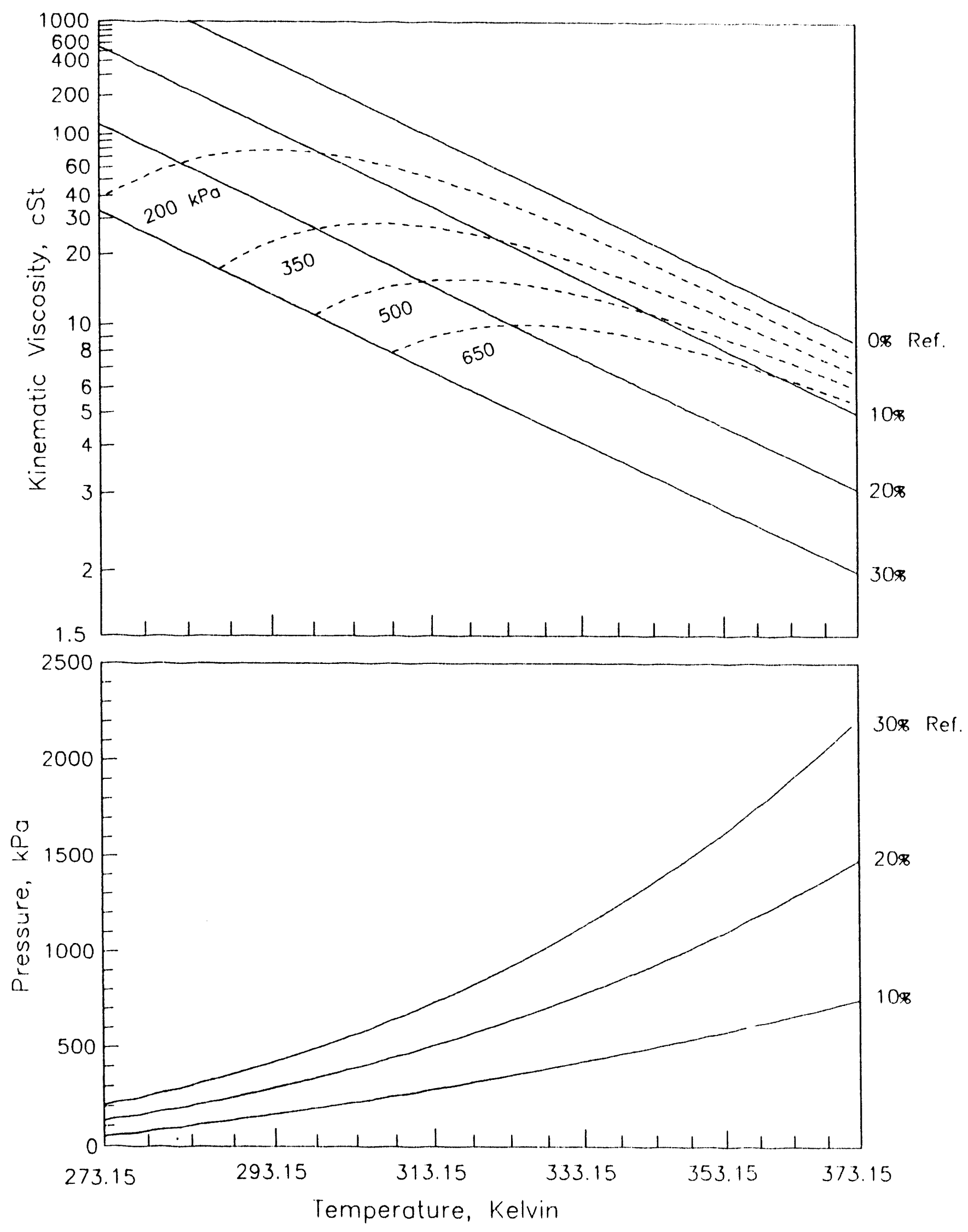


Table 3-1: Viscosity, Solubility and Density Parameters HCFC-22/ISO 32 Naphthenic Mineral Oil

$\begin{array}{cccc}\begin{array}{c}\text { Dynamic } \\ \text { Viscosity } \\ \text { (eq. 1) }\end{array} & \begin{array}{c}\text { Vapor } \\ \text { Pressure } \\ \text { (eq. 2) }\end{array} & \begin{array}{c}\text { Denisty } \\ \text { (eq. 3) }\end{array} & \begin{array}{c}\text { Kinematic } \\ \text { Viscosity } \\ \text { (eq. 4) }\end{array} \\ 144957 \mathrm{E}+1 & -3.435225 \mathrm{E}+3 & 1.091957 & 1.087016 \mathrm{E}+1 \\ -4.523308 & 2.124994 \mathrm{E}+1 & -6.323417 \mathrm{E}-4 & -4.285019 \\ -8.316656 & -3.198259 \mathrm{E}-2 & 5.955585 \mathrm{E}-1 & -7.602685 \\ 2.770585 & 4.652129 \mathrm{E}+4 & -1.031991 \mathrm{E}-3 & 2.488802 \\ 7.646033 & -3.657045 \mathrm{E}+2 & -2.339745 \mathrm{E}-1 & 8.483115 \\ -2.981429 & 7.362473 \mathrm{E}-1 & 9.920486 \mathrm{E}-4 & -3.382609 \\ 0.9992 & 0.9965 & 0.9999 & 0.9992\end{array}$

Figure 3-1: Density of HCFC-22/ISO 32 Naphthenic Mineral Oil

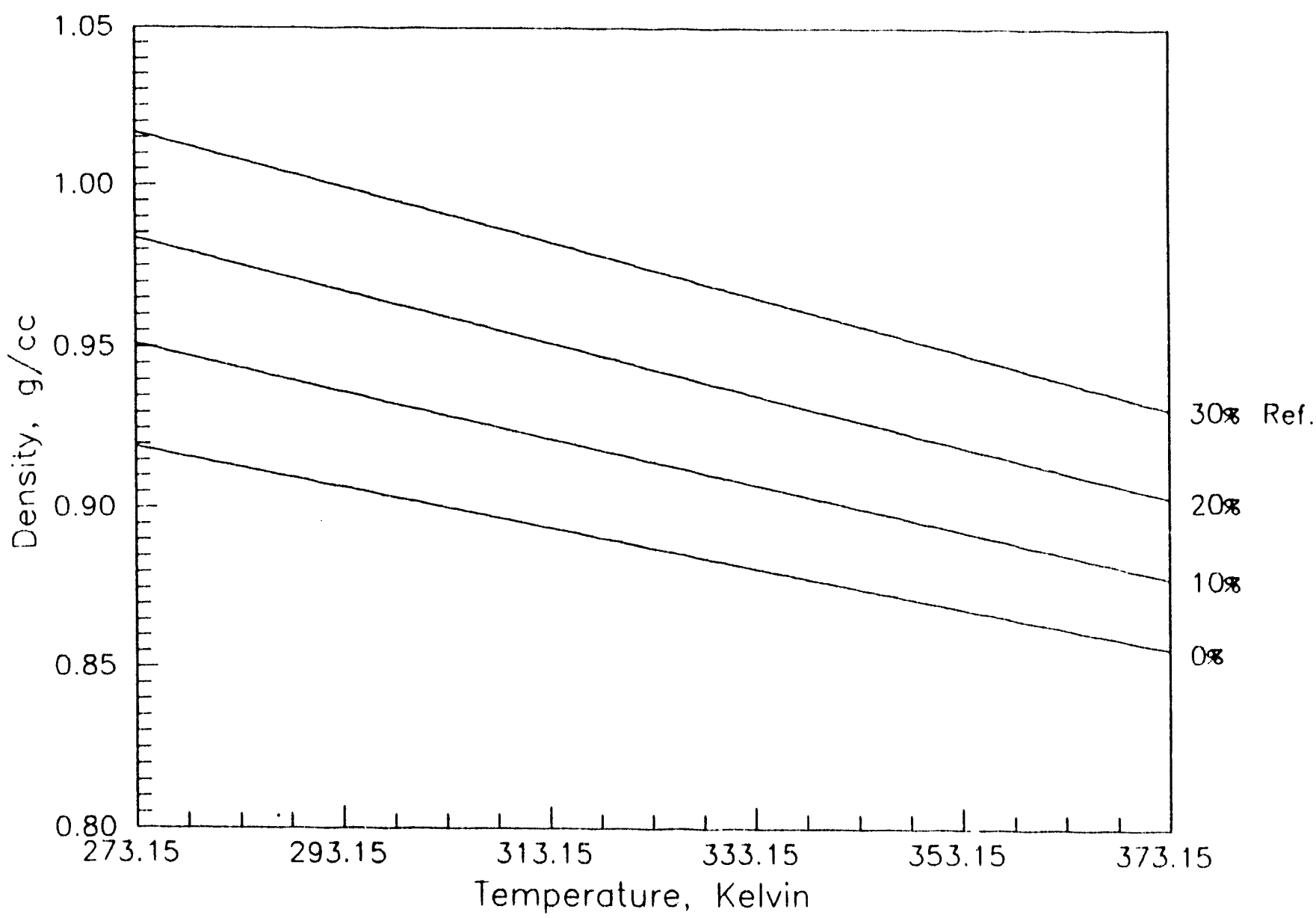


Figure 3-2: Viscosity and Solubility of HCFC-22/ISO 32 Naphthenic Mineral Oil

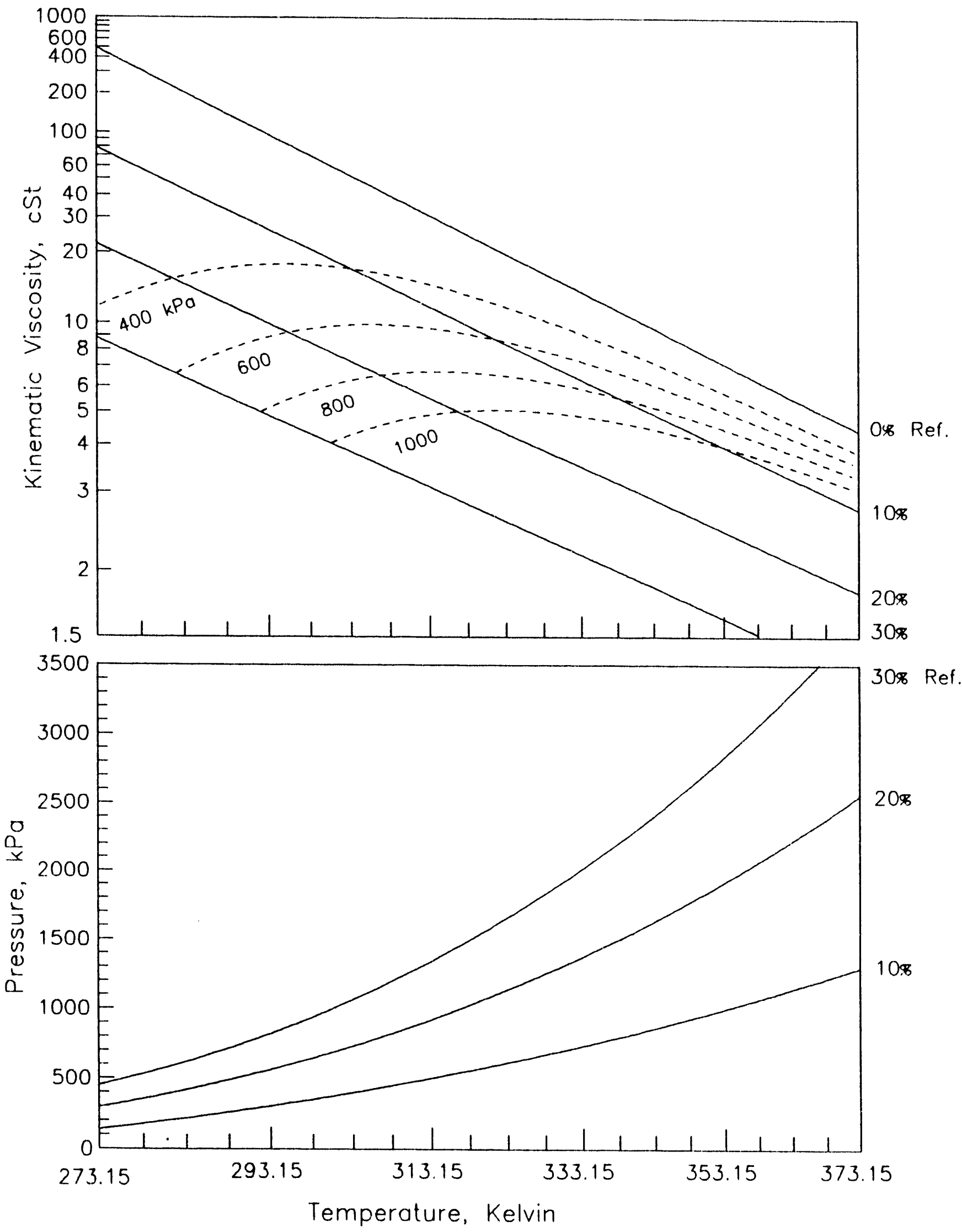


Table 5-1: Viscosity, Solubility and Density Parameters HFC-134a/ISO 22 Pentaerythritol Ester Mixed Acid

$\begin{array}{cccc}\text { Dynamic } & \text { Vapor } & & \text { Kinematic } \\ \text { Viscosity } & \text { Pressure } & \text { Density } & \text { Viscosity } \\ \text { (eq. 1) } & \text { (eq. 2) } & \text { (eq. 3) } & \text { (eq. 4) }\end{array}$

$\begin{array}{lcccc}a_{1} & 9.653926 & 1.470761 \mathrm{E}+3 & 1.210694 & 9.348190 \\ a_{2} & -3.815837 & -9.660980 & -7.453571 \mathrm{E}-4 & -3.691665 \\ \mathrm{a}_{3} & -7.712563 & 1.616140 \mathrm{E}-2 & 4.387843 \mathrm{E}-1 & -7.489899 \\ \mathrm{a}_{4} & 2.752815 & 3.222871 \mathrm{E}+4 & -7.157755 \mathrm{E}-4 & 2.656155 \\ \mathrm{a}_{3} & 2.182599 \mathrm{E}+1 & -2.646436 \mathrm{E}+2 & 3.688504 \mathrm{E}-1 & 2.240309 \mathrm{E}+1 \\ \mathrm{a}_{6} & -8.939830 & 5.445102 \mathrm{E}-1 & -1.212673 \mathrm{E}-3 & -9.206934 \\ \sigma & 0.9997 & 0.9959 & 0.9998 & 0.9997\end{array}$

Figure 5-1: Density of HFC-134a/ISO 22 Pentaerythritol Ester Mixed Acid

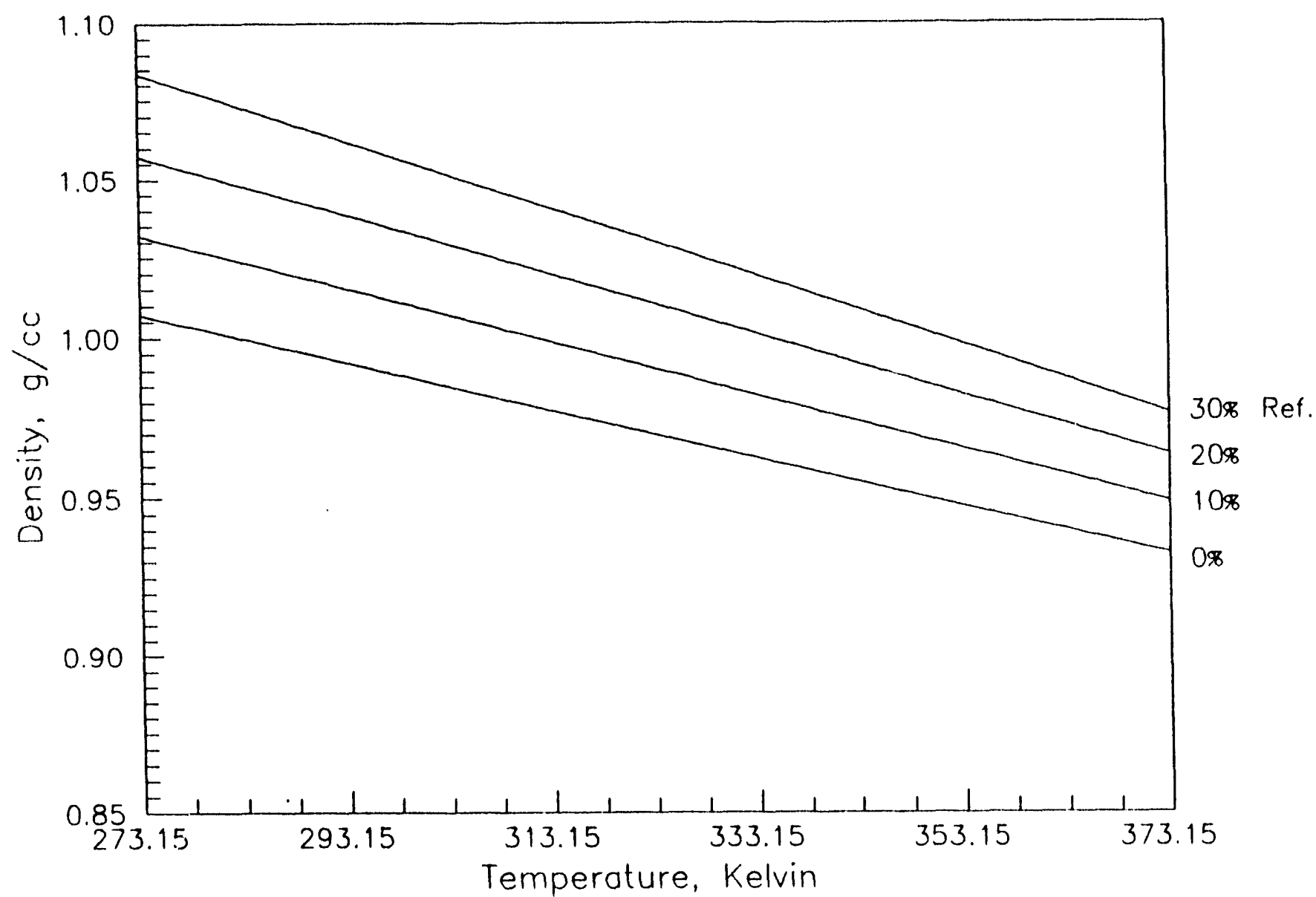


Figure 5-2: Viscosity and Solubility of HFC-134a/ISO 22 Pentaerythritol Ester Mixed Acid

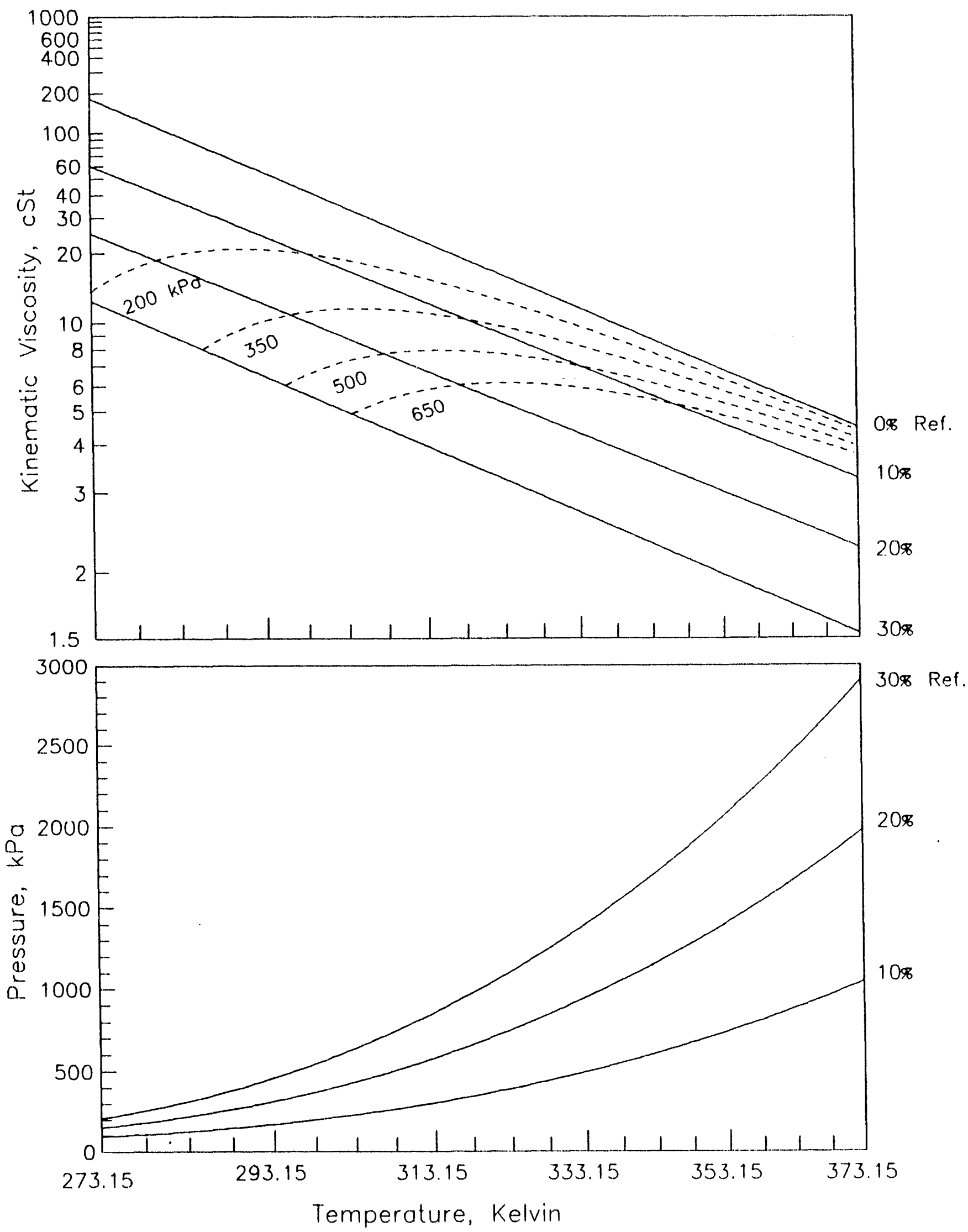


Table 6-1: Viscosity, Solubility and Density Parameters HFC-134a/ISO 32 Pentaerythritol Ester Mixed Acid \#1

Dynamic

(eq. 1)

$\begin{array}{cc}a_{1} & 9.928664 \\ a_{2} & -3.911169 \\ a_{3} & -1.100090 E+1 \\ a_{4} & 4.007297 \\ a_{5} & 3.371210 E+1 \\ a_{6} & -1.363471 E+1 \\ \sigma & 0.9997\end{array}$

Vapor

Pressure

(eq. 2)
Density

(eq. 3)
Kinematic

Viscosity

(eq. 4)

$\begin{array}{cc}-6.015270 \mathrm{E}+2 & 1.209498 \\ 3.333164 & -7.458734 \mathrm{E}-4 \\ -4.133328 \mathrm{E}-3 & 6.738057 \mathrm{E}-1 \\ 4.419037 \mathrm{E}+4 & -1.512800 \mathrm{E}-3 \\ -3.356229 \mathrm{E}+2 & -7.288073 \mathrm{E}-1 \\ 6.465144 \mathrm{E}-1 & 2.760360 \mathrm{E}-3 \\ 0.9965 & 0.9988\end{array}$

9.646453

$-3.796489$

$-1.148366 \mathrm{E}+1$

4.194896

$3.533470 \mathrm{E}+1$

$-1.432533$

0.9997

Figure 6-1: Density of HFC-134a/ISO 32 Pentaerythritol Ester Mixed Acid \#1

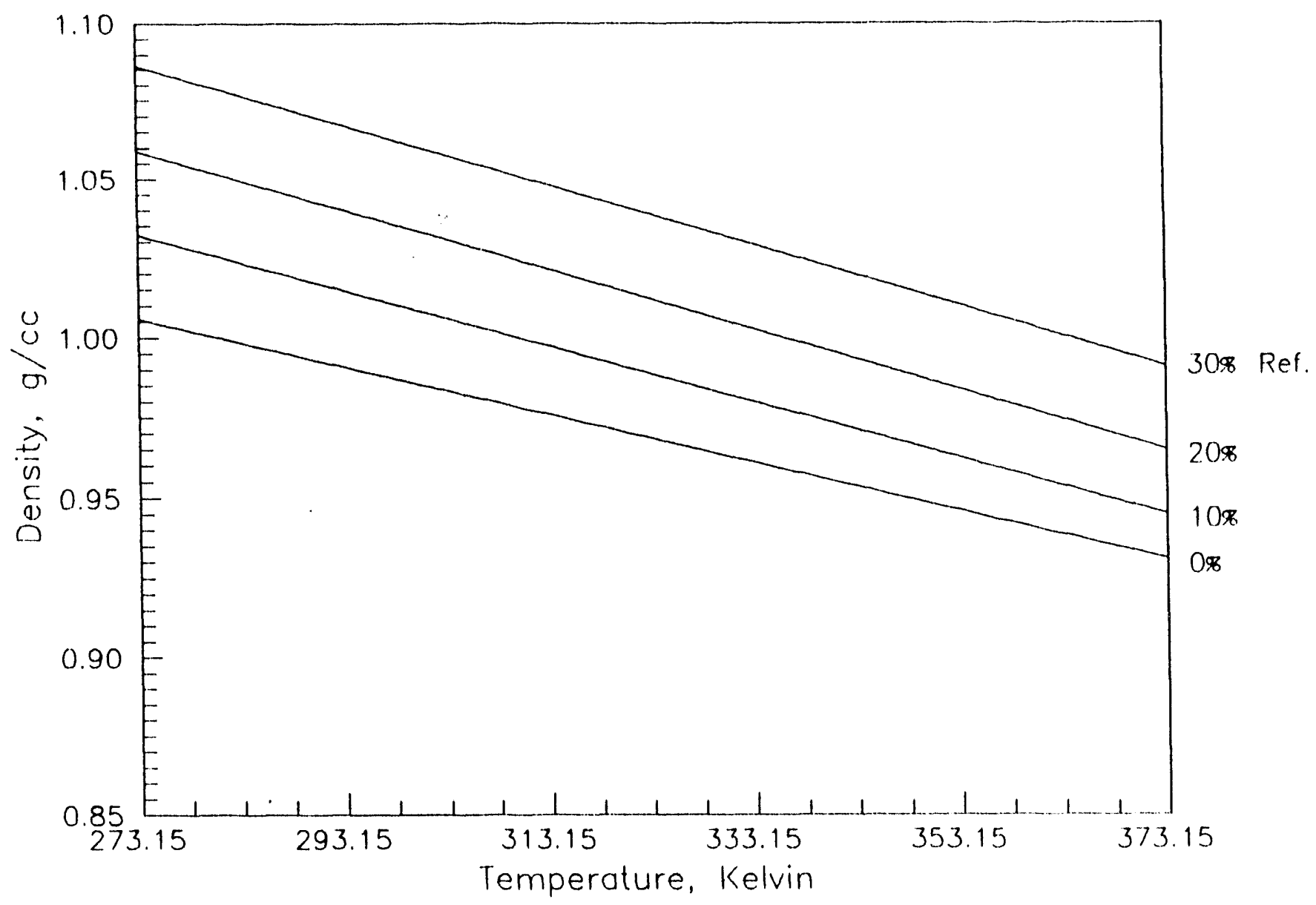


Figure 6-2: Viscosity and Solubility of IIFC-134a/ISO 32 Pentaerythritol Ester Mixed Acid \#1

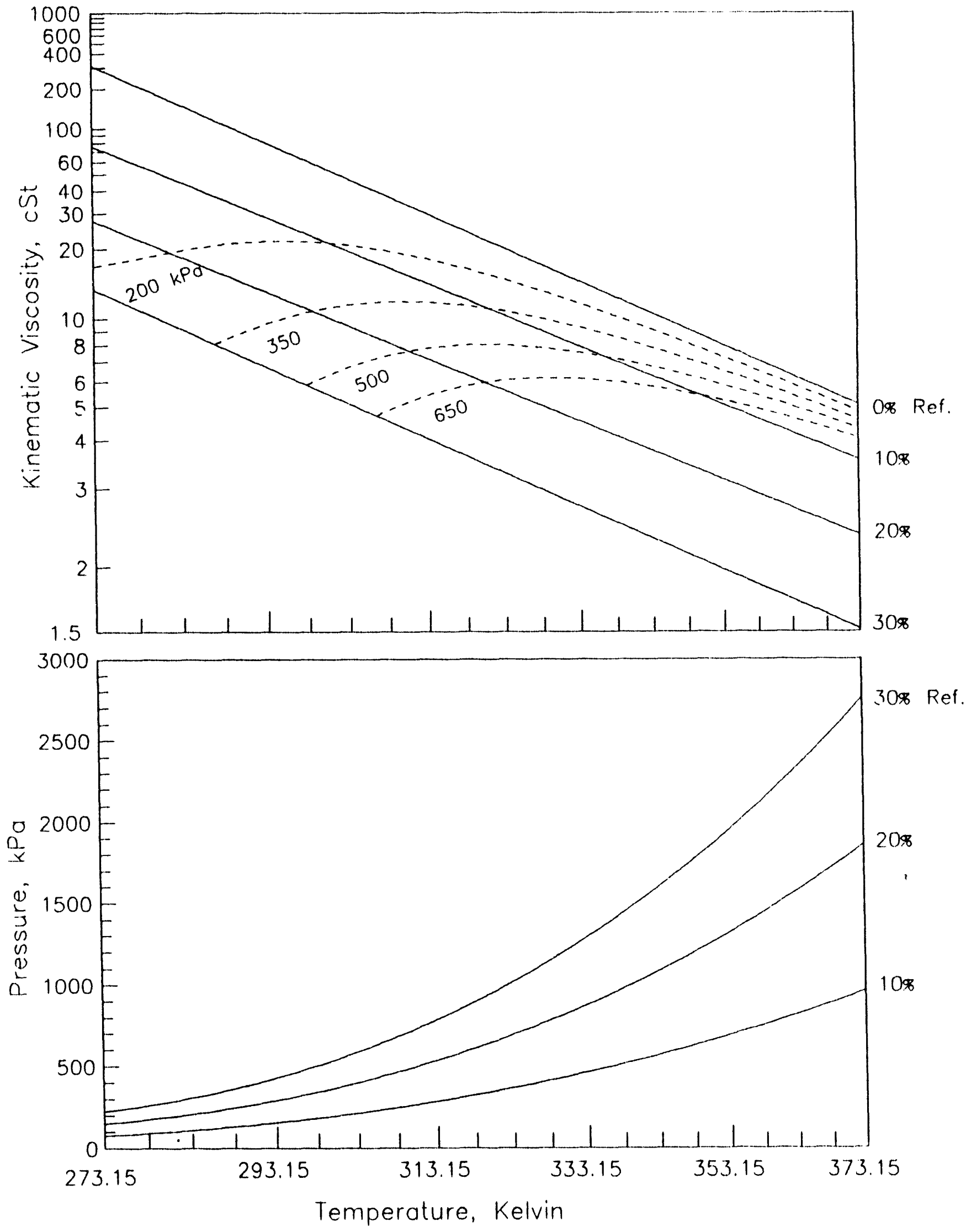


Table 7-1: Viscosity, Solubility and Density Parameters HFC-134a/ISO 68 Pentaerythritol Ester Mixed Acid

$\begin{array}{cccc}\text { Dynamic } & \text { Vapor } & & \text { Kinematic } \\ \text { Viscosity } & \text { Pressure } & \text { Density } & \text { Viscosity } \\ \text { (eq. 1) } & \text { (eq. 2) } & \text { (eq. 3) } & \text { (eq. 4) }\end{array}$

$\begin{array}{lcccc}a_{1} & 1.026622 \mathrm{E}+1 & -4.711721 \mathrm{E}+2 & 1.172826 & 9.926922 \\ a_{2} & -4.008826 & 2.419223 & -7.042171 \mathrm{E}-4 & -3.870717 \\ a_{3} & -2.183025 & -2.547792 \mathrm{E}-3 & 4.685648 \mathrm{E}-1 & -2.033556 \\ a_{4} & 3.281768 \mathrm{E}-1 & 4.563651 \mathrm{E}+4 & -6.414542 \mathrm{E}-4 & 2.670297 \mathrm{E}-1 \\ a_{5} & -7.161060 & -3.464107 \mathrm{E}+2 & 2.882264 \mathrm{E}-1 & -6.262914 \\ a_{6} & 3.147337 & 6.673004 \mathrm{E}-1 & -1.088702 \mathrm{E}-3 & 2.744884 \\ \sigma & 0.9995 & 0.9962 & 0.9998 & 0.9995\end{array}$

Figure 7-1: Density of HFC-134a/1SO 68 Pentaerythritol Ester Mixed Acid

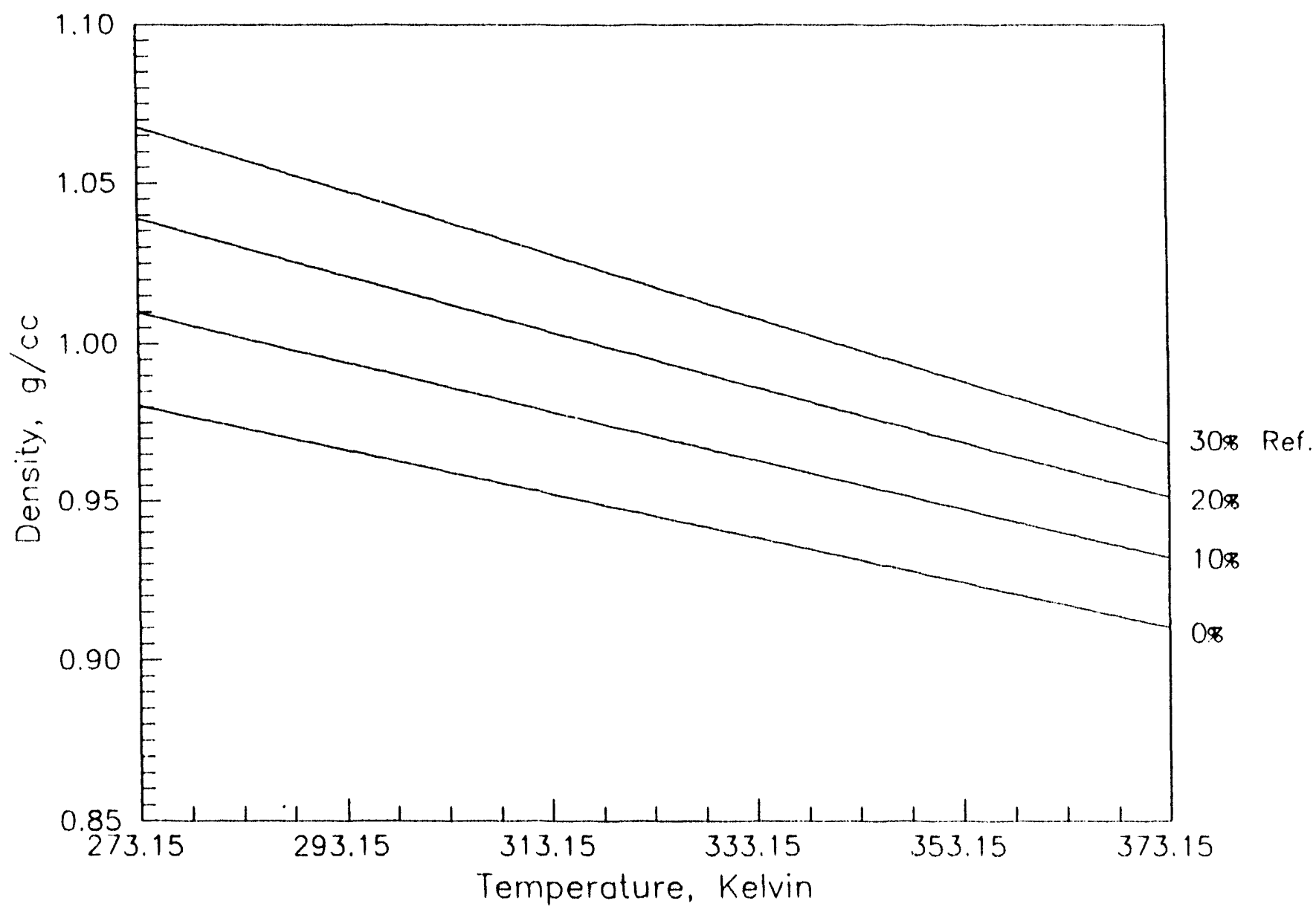


Figure 7-2: Viscosity and Solubility of HFC-134a/1SO 68 Pentaerythritol Ester Mixed Acid

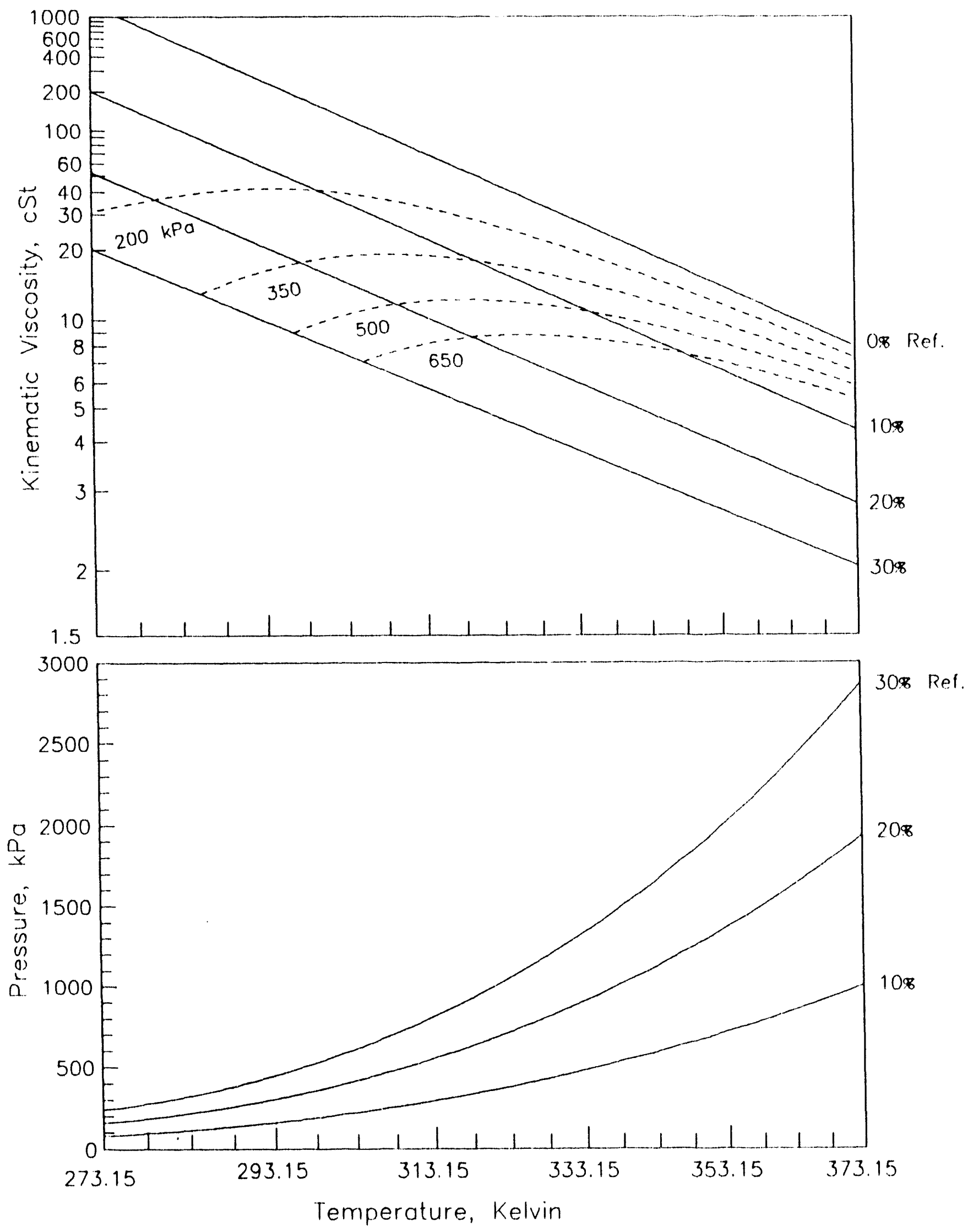


Table 8-1: Viscosity, Solubility and Density Parameters IIFC-134a/ISO 100 Pentaerythritol Ester Mixed Acid

$\begin{array}{cccc}\text { Dynamic } & \text { Vapor } & & \text { Kinematic } \\ \text { Viscosity } & \text { Pressure } & \text { Density } & \text { Viscosity } \\ \text { (eq. 1) } & \text { (eq. 2) } & \text { (eq. 3) } & \text { (eq. 4) }\end{array}$

$\begin{array}{lcccc}a_{1} & 9.011855 & -2.746753 E+3 & 1.174532 & 8.727968 \\ a_{2} & -3.494464 & 1.658969 E+1 & -6.917701 E-4 & -3.379039 \\ a_{3} & 7.903044 & -2.438650 E-2 & 6.085326 E-1 & 7.516009 \\ a_{4} & -3.610207 & 5.815944 E+4 & -1.214404 \mathrm{E}-3 & -3.453443 \\ a_{5} & -2.089983 \mathrm{E}+1 & -4.254682 \mathrm{E}+2 & -3.789143 \mathrm{E}-1 & -1.848345 \\ a_{6} & 8.268137 & 7.914398 \mathrm{E}-1 & 1.381617 \mathrm{E}-3 & 7.251276 \\ \sigma & 0.9993 & 0.9963 & 0.9997 & 0.9994\end{array}$

Figure 8-1: Density of HFC-134a/ISO 100 Pentaerythritol Ester Mixed Acid

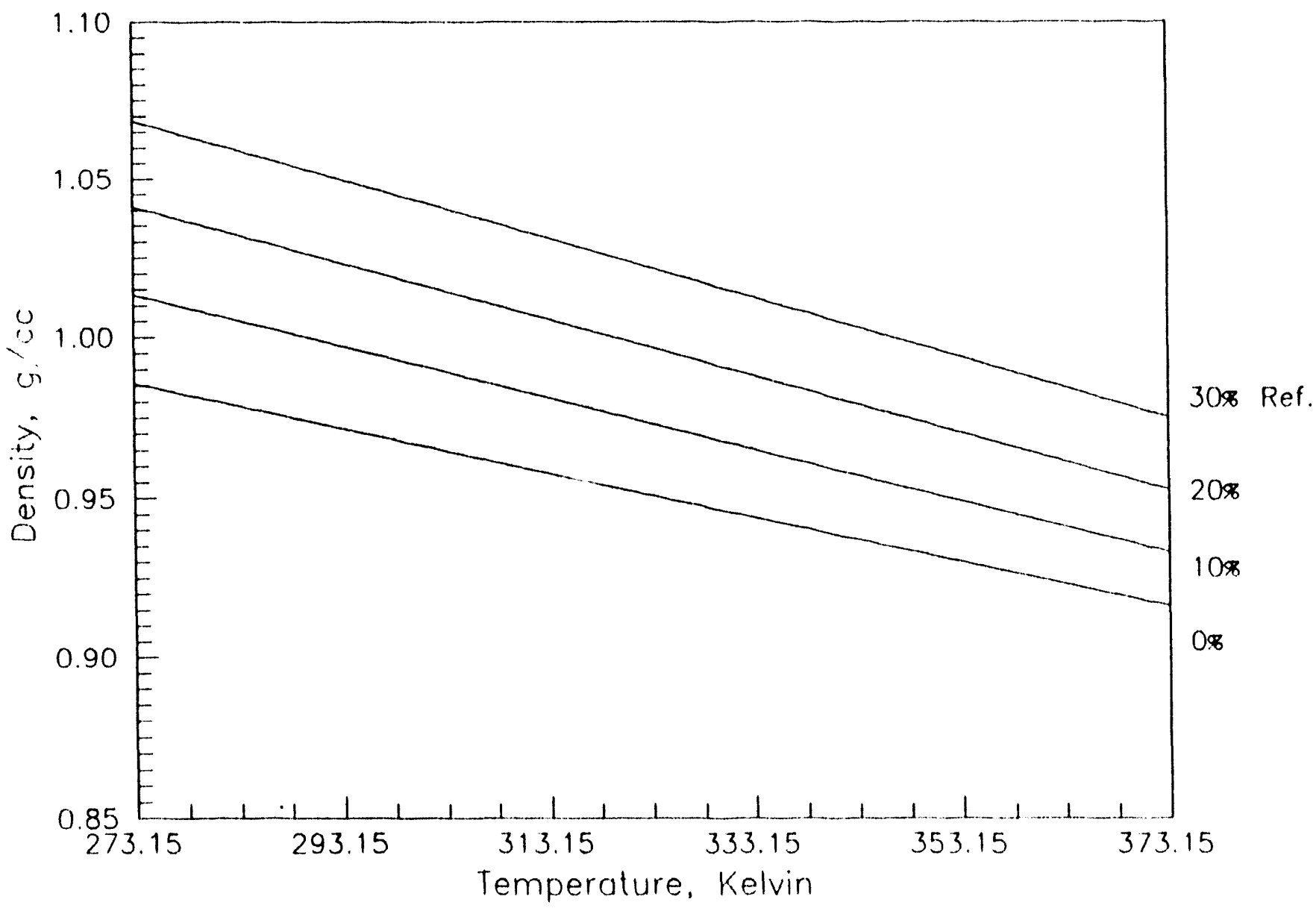


Figure 8-2: Viscosity and Solubility of HIFC-134a/ISO 100 Pentaerythritol Ester Mixed Acid

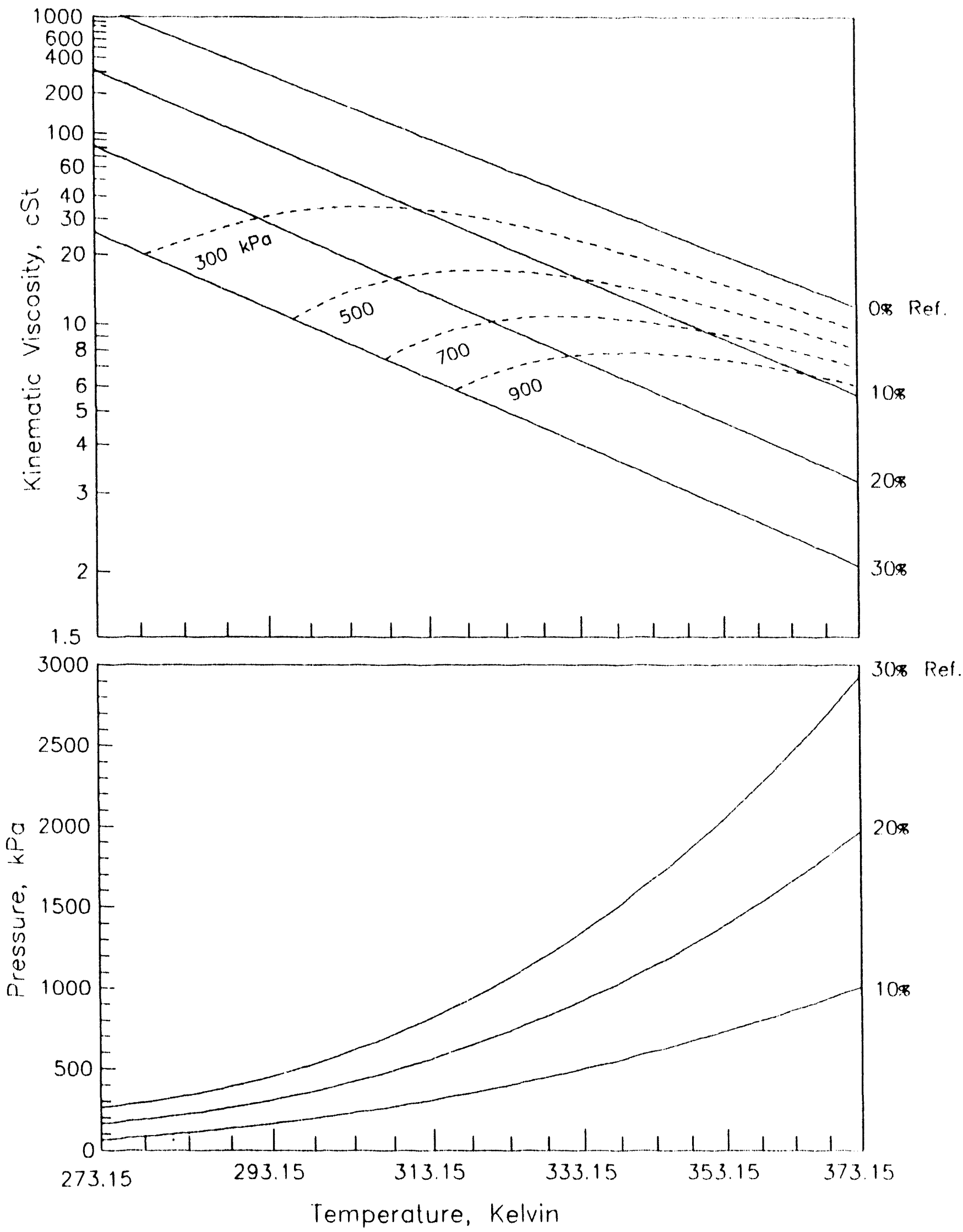


Table 10-1: Viscosity, Solubility and Density Parameters HFC-134a/ISO 32 Pentaerythritol Ester Mixed Acid \#2

$\begin{array}{cccc}\text { Dynamic } & \text { Vapor } & & \text { Kinematic } \\ \text { Viscosity } & \text { Pressure } & \text { Density } & \text { Viscosity } \\ \text { (eq. 1) } & \text { (eq. 2) } & \text { (eq. 3) } & \text { (eq. 4) }\end{array}$

$\begin{array}{lcccc}a_{1} & 9.249874 & -4.068101 \mathrm{E}+3 & 1.217296 & 8.982900 \\ a_{2} & -3.638505 & 2.546544 \mathrm{E}+1 & -7.504065 \mathrm{E}-4 & -3.530265 \\ a_{3} & 7.427879 & -3.944581 \mathrm{E}-2 & 6.014204 \mathrm{E}-1 & 7.052171 \\ a_{4} & -3.254920 & 5.191589 \mathrm{E}+4 & -1.223477 \mathrm{E}-3 & -3.109581 \\ a_{3} & -3.175478 \mathrm{E}+1 & -3.860092 \mathrm{E}+2 & -4.574697 \mathrm{E}-1 & -2.872141 \mathrm{E}+1 \\ a_{6} & 1.226973 \mathrm{E}+1 & 7.284821 \mathrm{E}-1 & 1.599145 \mathrm{E}-3 & 1.100634 \mathrm{E}+1 \\ \sigma & 0.9996 & 0.9955 & 0.9997 & 0.9995\end{array}$

Figure 10-1: Density of IIFC-134a/ISO 32 Pentaerythritol Ester Mixed Acid \#2

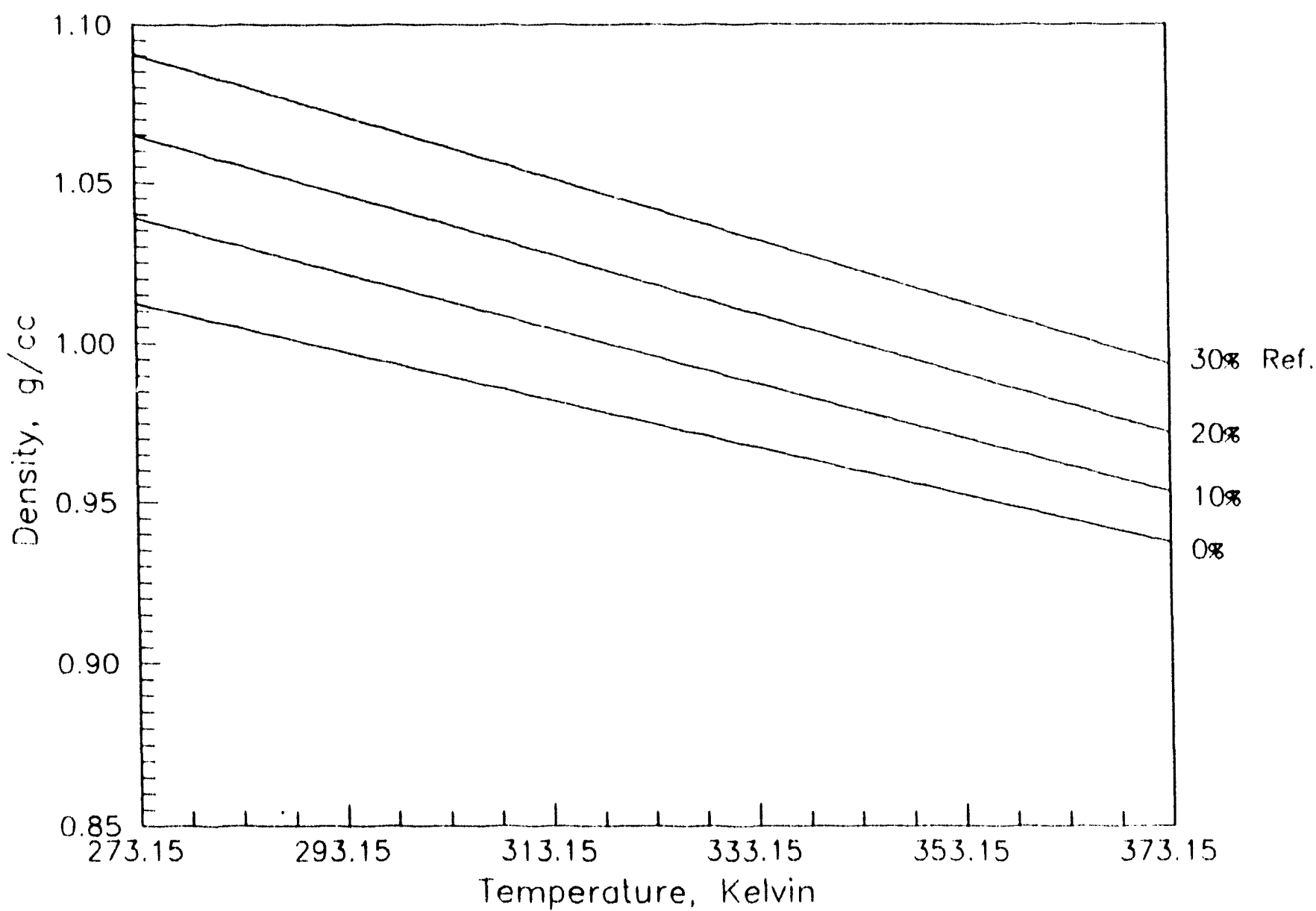


Figure 10-2: Viscosity and Solubility of HFC-134a/1SO 32 Pentaerythritol Ester Mixed Acid \#2

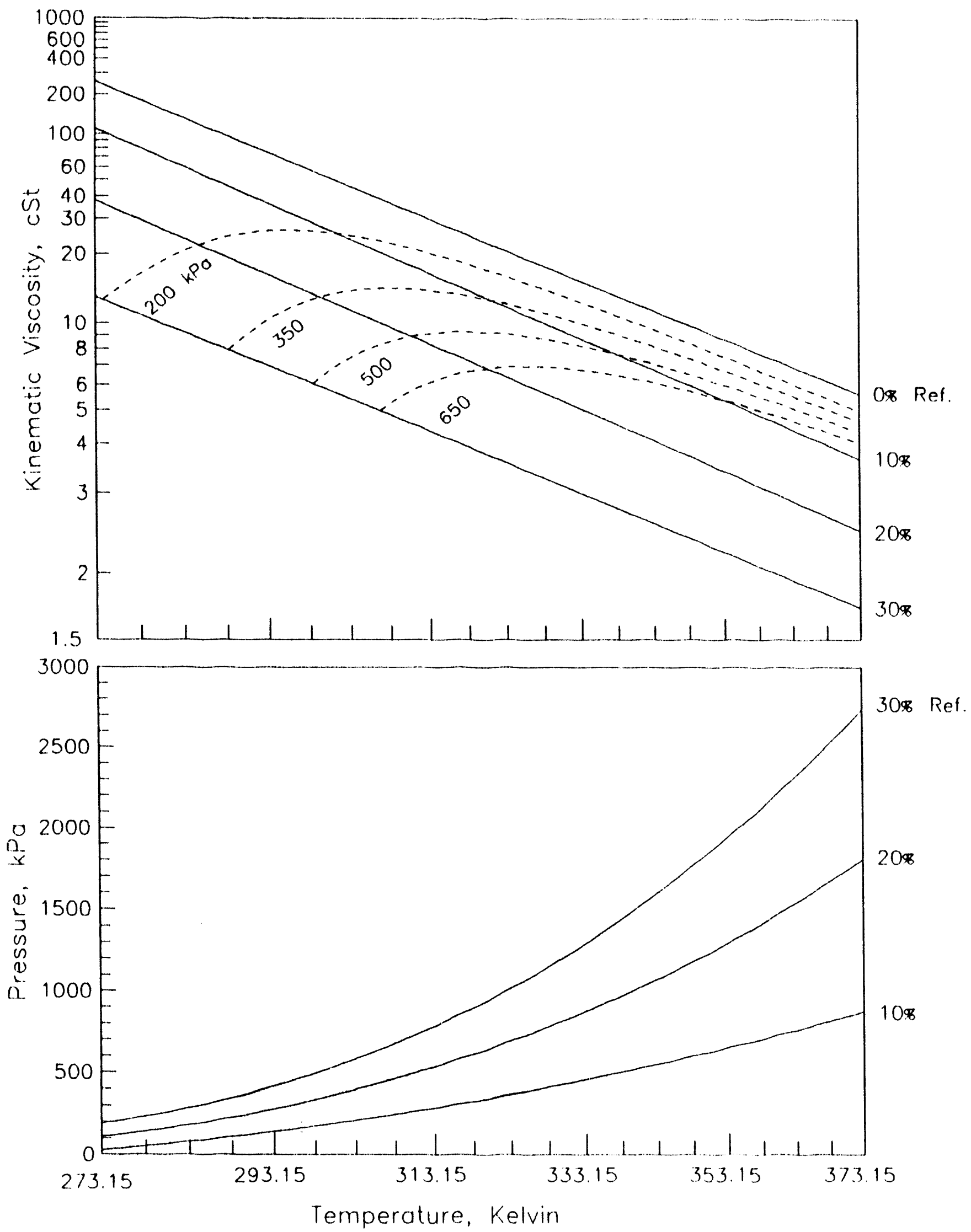


Table 13-1: Viscosity, Solubility and Density Parameters HCFC-123/ISO 32 Naphthenic Mineral Oil

Dynamic

Viscosity

(eq. 1)

$\begin{array}{lcc}a_{1} & 1.143884 \mathrm{E}+1 & 1.645131 \mathrm{E}+1 \\ \mathrm{a}_{2} & -4.519219 & -1.805161 \mathrm{E}-1 \\ \mathrm{a}_{3} & -1.294323 & 4.648786 \mathrm{E}-4 \\ \mathrm{a}_{4} & 2.134015 \mathrm{E}-1 & 1.091676 \mathrm{E}+4 \\ \mathrm{a}_{5} & -5.988494 & -7.757201 \mathrm{E}+1 \\ \mathrm{a}_{6} & 2.336175 & 1.399709 \mathrm{E}-1 \\ \sigma & 0.9994 & 0.9965\end{array}$

Vapor

Pressure

(eq. 2)
Density

(eq. 3)
Kinematic

Viscosity

(eq. 4)

$\begin{array}{cc}1.091381 & 1.086147 \mathrm{E}+1 \\ -6.333717 \mathrm{E}-4 & -4.281662 \\ 7.350596 \mathrm{E}-1 & -1.499504 \mathrm{E}-1 \\ -8.552796 \mathrm{E}-4 & -2.615068 \mathrm{E}-1 \\ -4.946925 \mathrm{E}-1 & -6.104743 \\ 3.170175 \mathrm{E}-4 & 2.372308 \\ 0.9984 & 0.9992\end{array}$

Figure 13-1: Density of HCFC-123/1SO 32 Naphthenic Mineral Oil

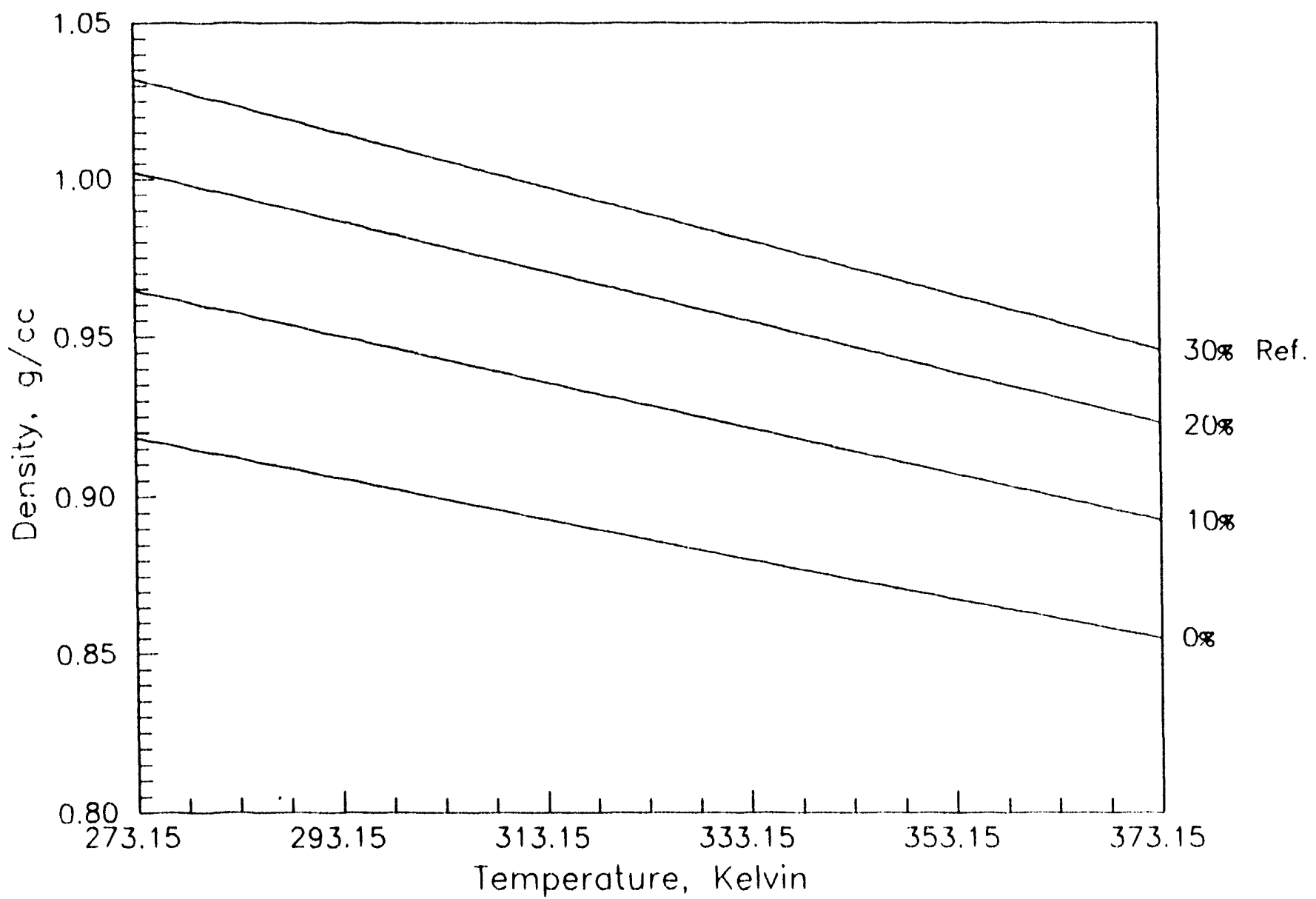


Figure 13-2: Viscosity and Solubility of HCFC-123/1SO 32 Naphthenic Mineral Oil

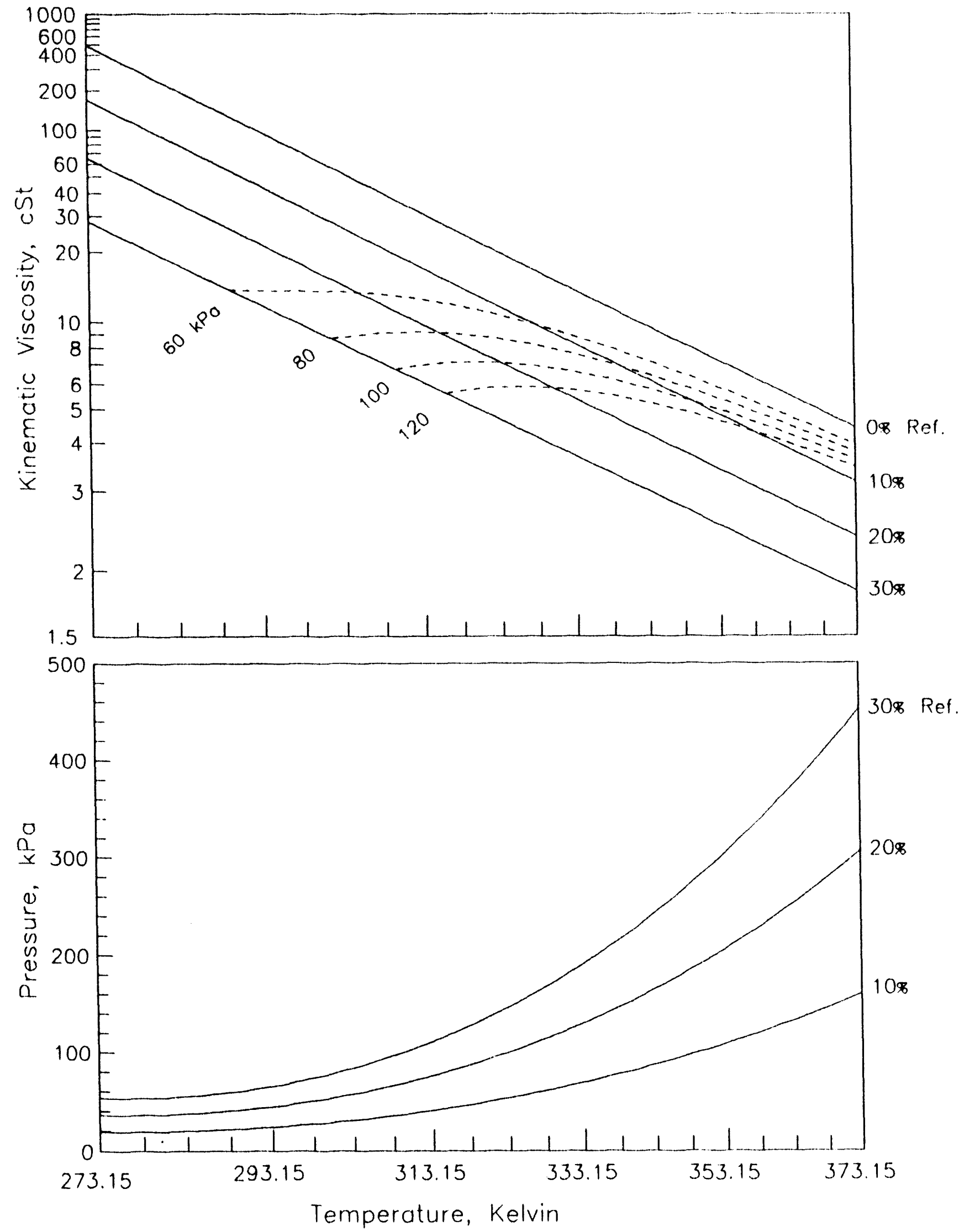


Table 14-1: Viscosity, Solubility and Density Parameters HCFC-123/ISO 100 Naphthenic Mineral Oil

Dynamic

Viscosity

(eq. 1)

$\begin{array}{lc}\mathrm{a}_{1} & 1.167333 \mathrm{E}+1 \\ \mathrm{a}_{2} & -4.319983 \\ \mathrm{a}_{3} & 4.689200 \\ \mathrm{a}_{4} & -2.189975 \\ \mathrm{a}_{3} & -2.852759 \mathrm{E}+1 \\ \mathrm{a}_{6} & 1.130683 \mathrm{E}+1 \\ \sigma & 0.9993\end{array}$

Vapor

Pressure

(eq. 2)
Density

(eq. 3)
Kinematic

Viscosity

(eq. 4)

$$
\begin{gathered}
-2.437128 \mathrm{E}+2 \\
1.329632 \\
-1.674724 \mathrm{E}-3 \\
1.305223 \mathrm{E}+4 \\
-9.146282 \mathrm{E}+1 \\
1.623400 \mathrm{E}-1 \\
0.9941
\end{gathered}
$$

1.097063 $-6.249877 \mathrm{E}-4$

$1.060366 \mathrm{E}+1$

$5.919157 \mathrm{E}-1$

$-8.171586 \mathrm{E}-4$

$-4.127783$

$2.315816 \mathrm{E}-1$

$-3.002284 \mathrm{E}-4$

0.9996

4.365817

$-2.064418$

$-2.460633 \mathrm{E}+1$

9.697289

0.9993

Figure 14-1: Density of HCFC-123/1SO 100 Naphthenic Mineral Oil

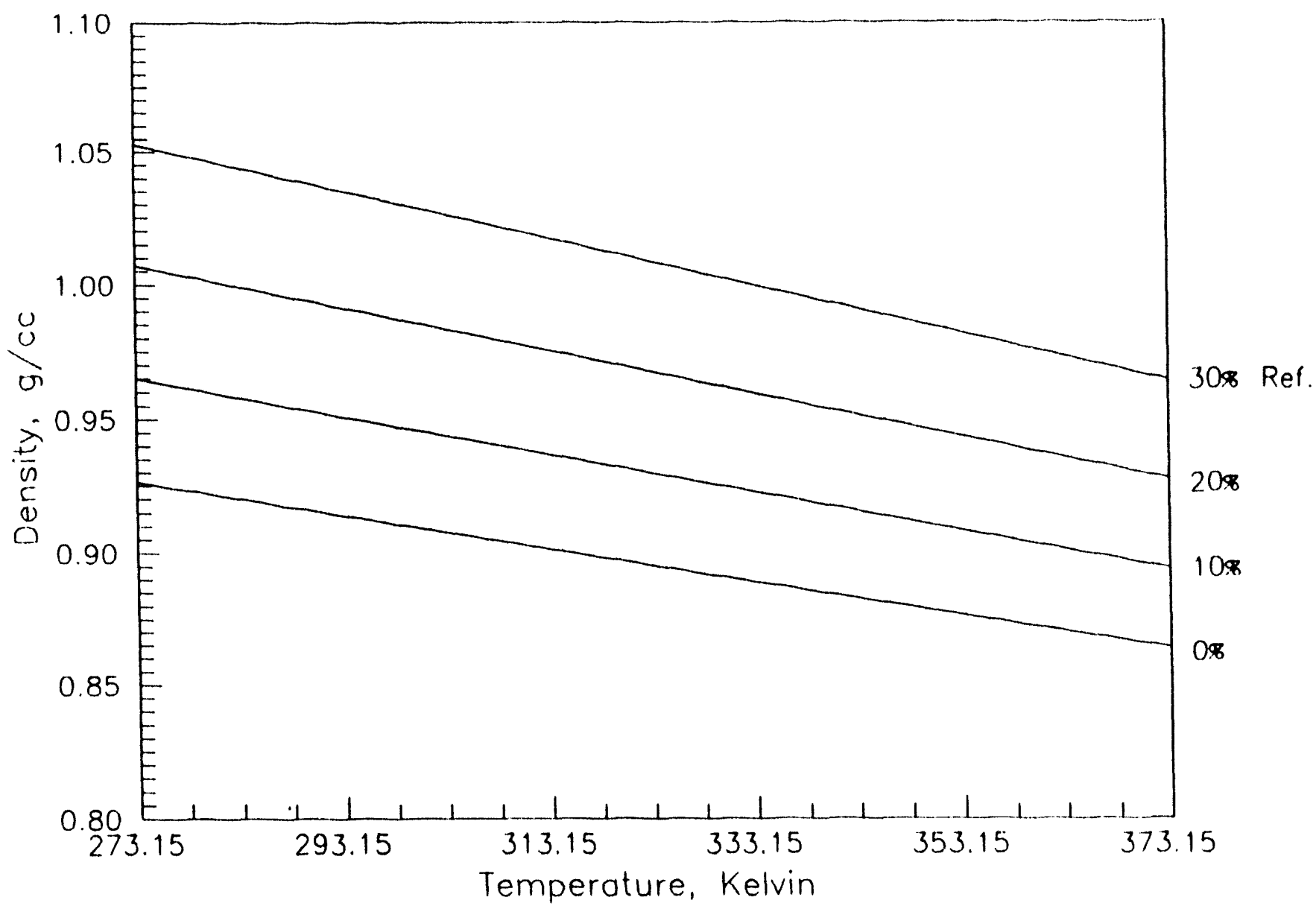


Figure 14-2: Viscosity and Solubility of IICFC-123/ISO 100 Naphthenic Mineral Oil

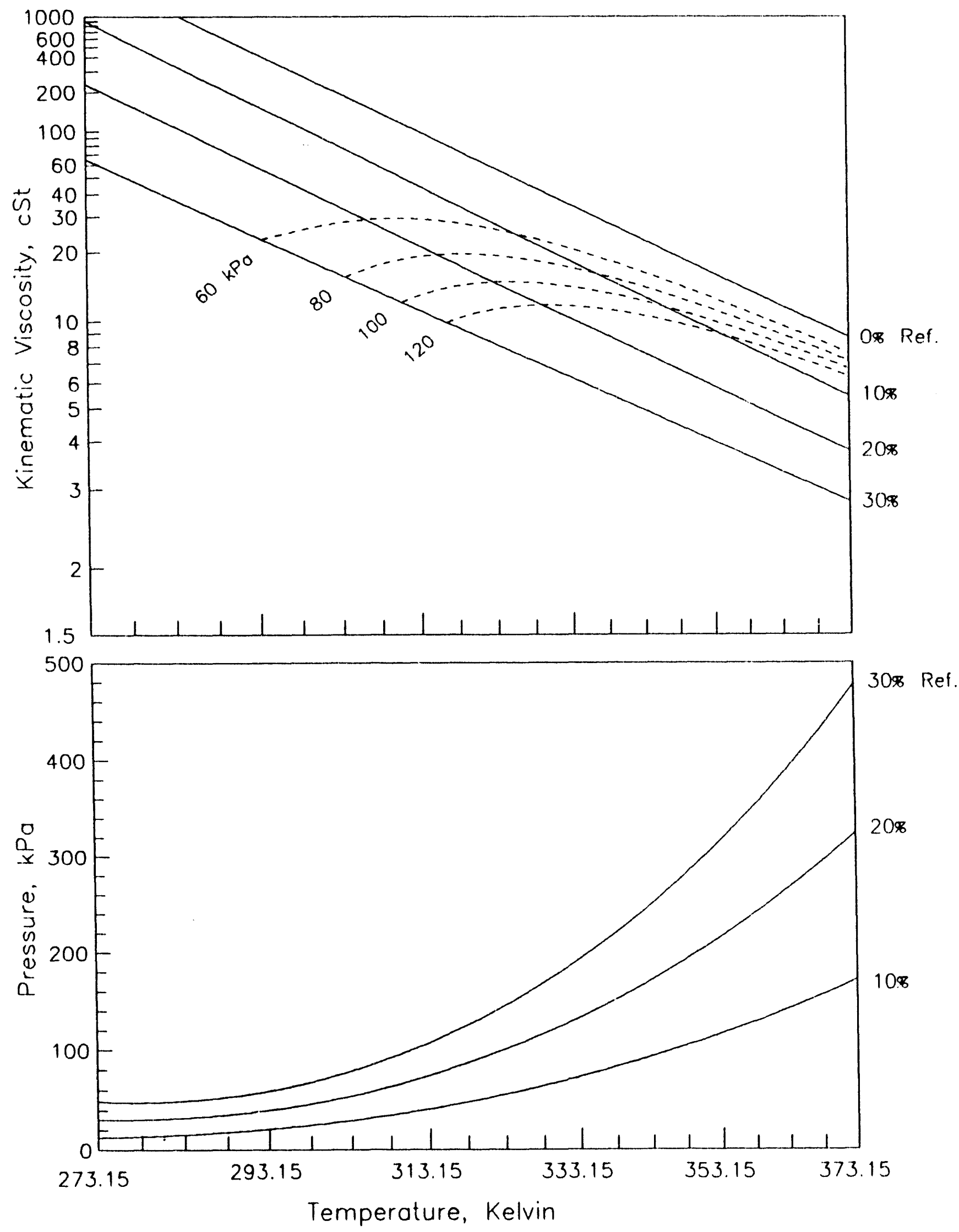


Table 15-1: Viscosity, Solubility and Density Parameters HCFC-123/150 SUS Alkylbenzene

$\begin{array}{cccc}\text { Dynamic } & \text { Vapor } & & \text { Kinematic } \\ \text { Viscosity } & \text { Pressure } & \text { Density } & \text { Viscosity } \\ \text { (eq. 1) } & \text { (eq. 2) } & \text { (eq. 3) } & \text { (eq. 4) }\end{array}$

$\begin{array}{lcccc}\mathrm{a}_{1} & 1.159926 \mathrm{E}+1 & -2.751188 \mathrm{E}+2 & 1.059407 & 1.085811 \mathrm{E}+1 \\ \mathrm{a}_{2} & -4.590598 & 1.644120 & -6.522680 \mathrm{E}-4 & -4.284822 \\ \mathrm{a}_{3} & -8.912714 & -2.359617 \mathrm{E}-3 & 5.309700 \mathrm{E}-1 & -7.213424 \\ \mathrm{a}_{4} & 3.274382 & 1.054293 \mathrm{E}+4 & -5.543972 \mathrm{E}-4 & 2.585382 \\ \mathrm{a}_{5} & 3.228156 \mathrm{E}+1 & -7.440249 & 3.925389 \mathrm{E}-1 & 3.088392 \mathrm{E}+1 \\ \mathrm{a}_{6} & -1.309060 \mathrm{E}+1 & 1.326417 \mathrm{E}-1 & -7.718499 \mathrm{E}-4 & -1.257611 \\ \sigma & 0.9987 & 0.9951 & 0.9999 & 0.9987\end{array}$

Figure 15-1: Density of HCFC-123/150 SUS Alkylbenzene

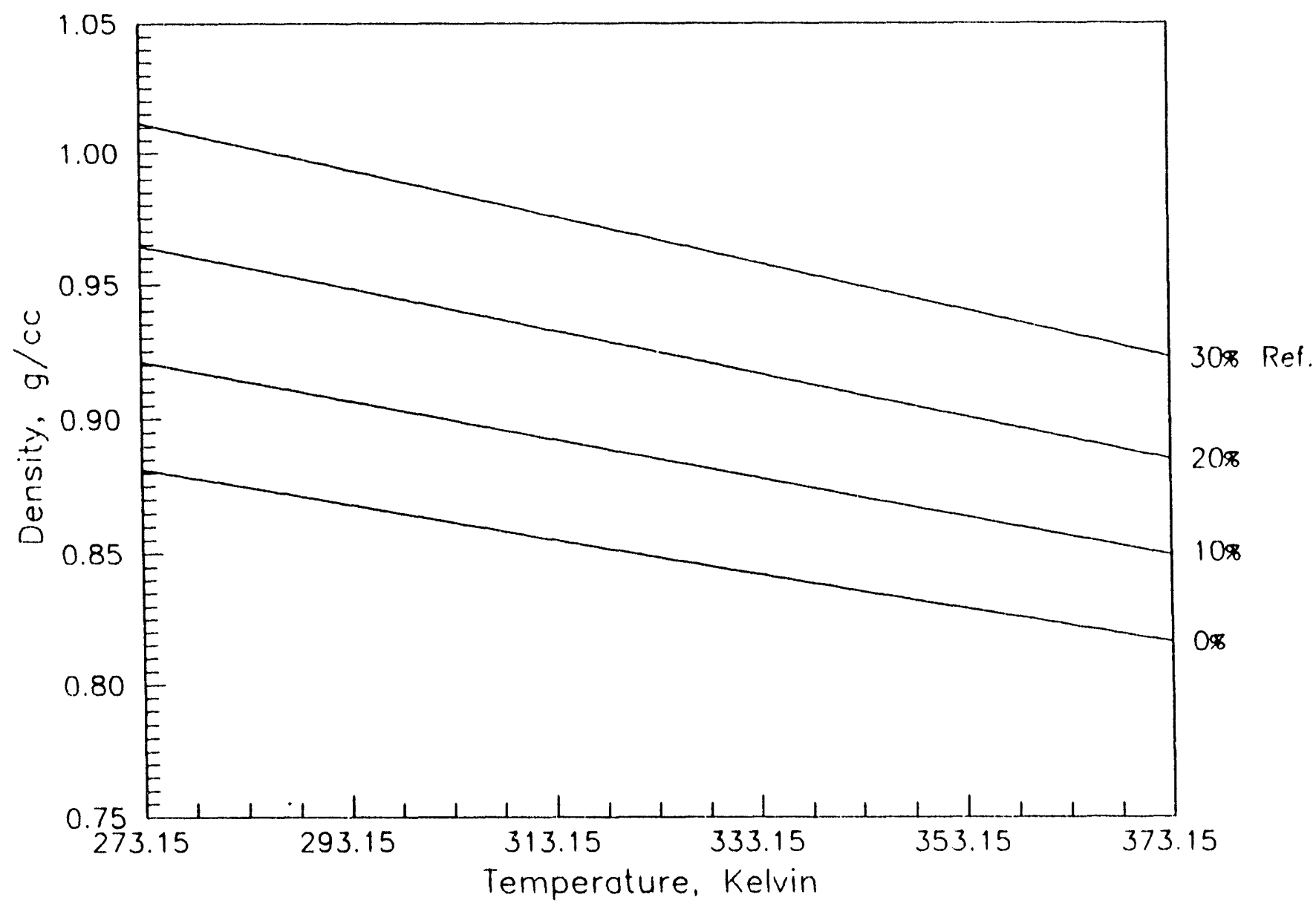


Figure 15-2: Viscosity and Solubility of HCFC-123/150 SUS Alkylbenzene
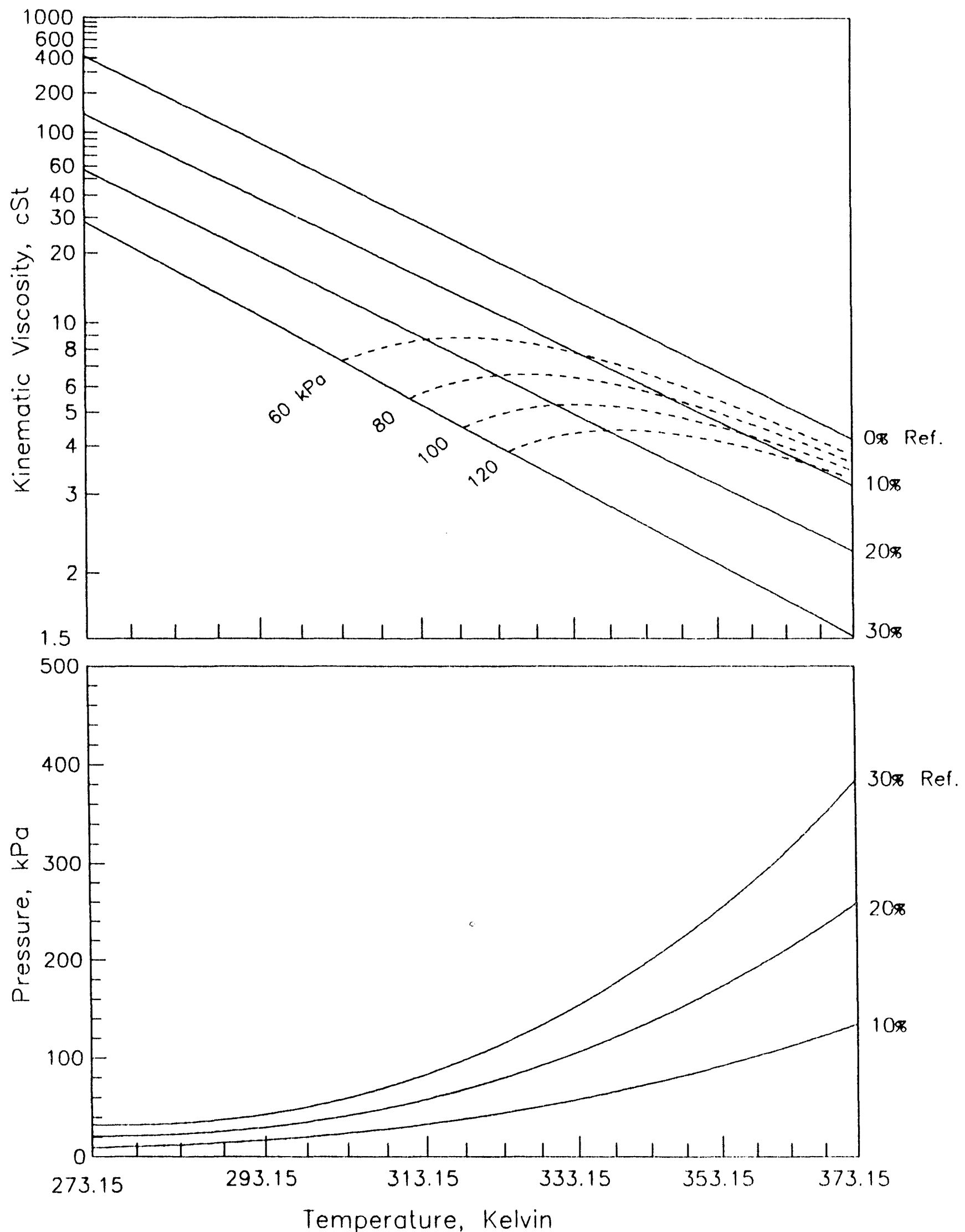
Table 16-1: Viscosity, Solubility and Density Parameters HCFC-123/300 SUS Alkylbenzene

$\begin{array}{lcccc}\begin{array}{c}\text { Dynamic } \\ \text { Viscosity } \\ \text { (eq. 1) }\end{array} & \begin{array}{c}\text { Vapor } \\ \text { Pressure } \\ \text { (eq. 2) }\end{array} & \begin{array}{c}\text { Density } \\ \text { (eq. 3) }\end{array} & \begin{array}{c}\text { Kinematic } \\ \text { Viscosity } \\ \text { (eq. 4) }\end{array} \\ \mathrm{a}_{1} & 1.193427 \mathrm{E}+1 & 1.365282 \mathrm{E}+2 & 1.055710 & 1.120515 \mathrm{E}+1 \\ \mathrm{a}_{2} & -4.694749 & -9.185995 \mathrm{E}-1 & -6.409276 \mathrm{E}-4 & -4.395374 \\ \mathrm{a}_{3} & -3.572445 & 1.584146 \mathrm{E}-3 & 5.646018 \mathrm{E}-1 & -2.616876 \\ \mathrm{a}_{4} & 1.109628 & \mathbf{8 . 6 3 8 0 1 5 \mathrm { E } + 3} & -7.023060 \mathrm{E}-4 & 7.280079 \mathrm{E}-1 \\ \mathrm{a}_{5} & 7.504941 & -6.294055 & 2.771386 \mathrm{E}-1 & 8.686595 \\ \mathrm{a}_{6} & -3.168215 & 1.157114 \mathrm{E}-1 & -3.084357 \mathrm{E}-4 & -3.7001282 \\ \sigma & 0.9995 & 0.9969 & 0.9999 & 0.9994\end{array}$

Figure 16-1: Density of HCFC-123/300 SUS Alkylbenzene

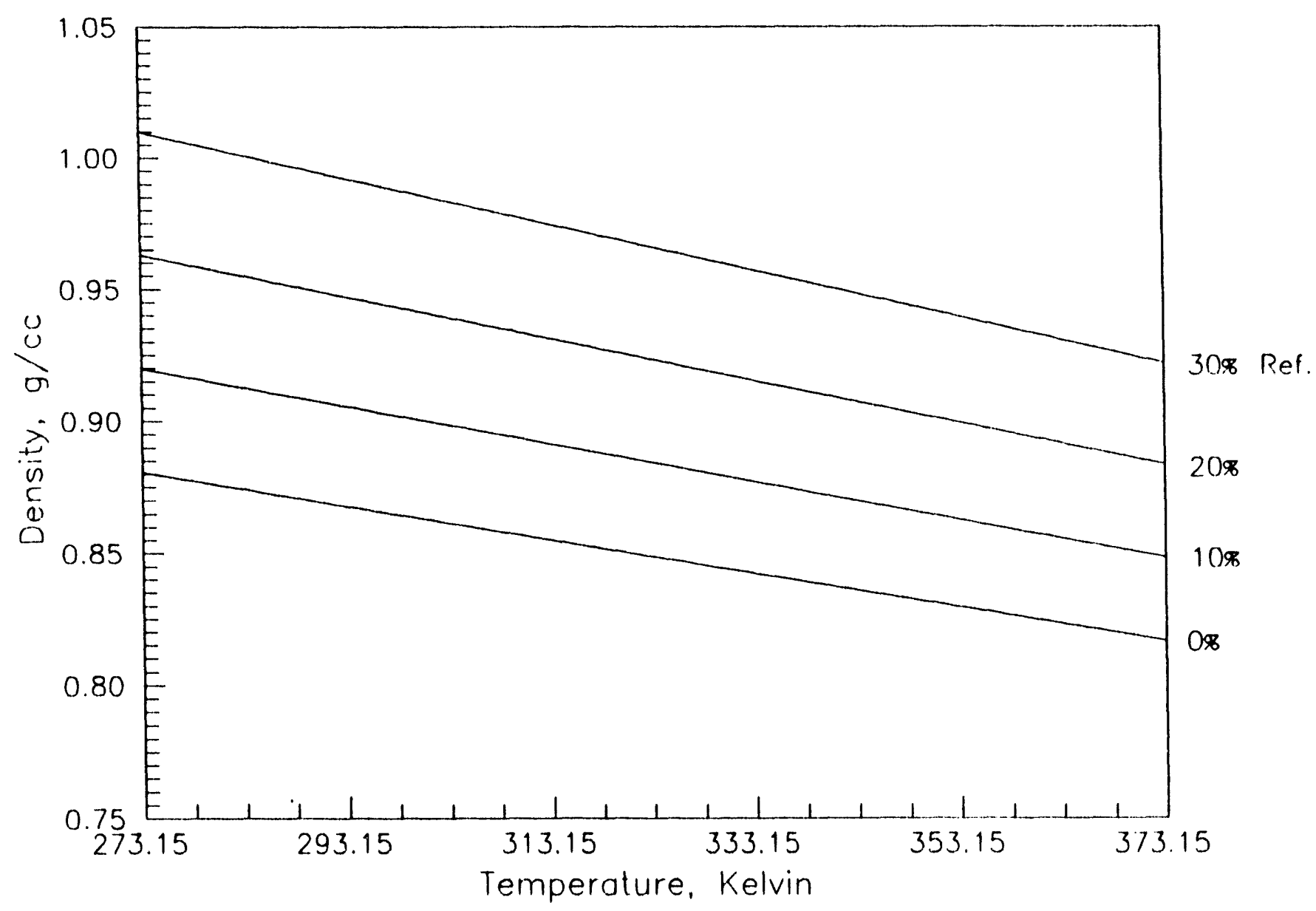


Figure 16-2: Viscosity and Solubility of HCFC-123/300 SUS Alkylbenzene

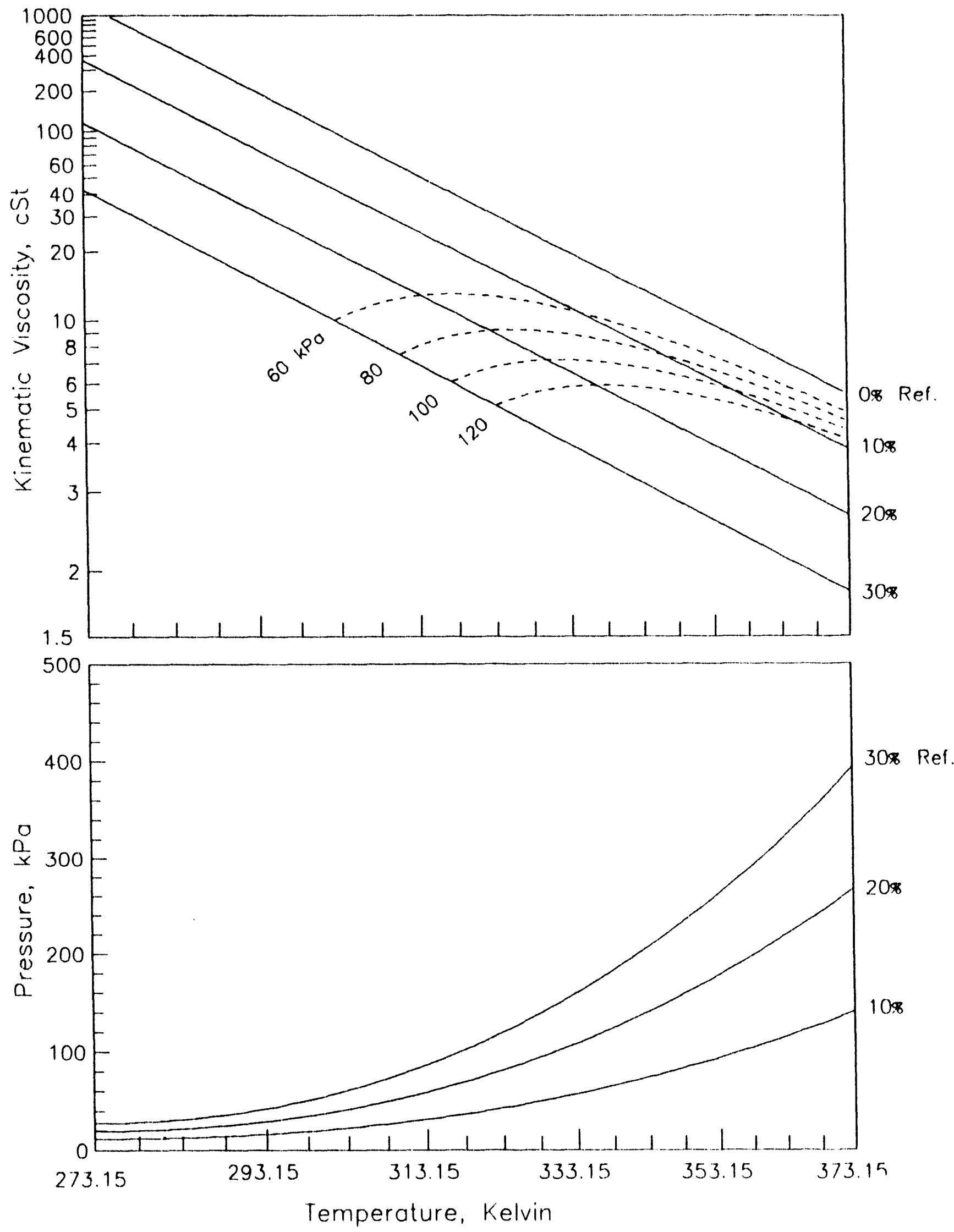




\section{COMPLIANCE WITILAGREEMENT}

No significant modifications or deviations from the technical performance of work as described in the contract agreement have been necessary during this reporting period.

\section{PRINCIPAL INVESTIGATOR EFFORT}

During this reporting period, Mr. David R. Henderson directed and/or participated in the following activities:

- Response to requests from ARTI involving discrepancies among contractors

- Project management and laboratory supervision

- Data reduction/mathematical modeling

- Reporting 


\section{APPENDIX A}

\section{Corrections for Vapor Space Volume in Test Apparatus}

The theoretical basis for the following development lies in application of the Gibbs Phase Rule, which will show that the two component fluid systems under study here, for all practical purposes, have two degrees of freedom. Thus, if temperature and density in one vessel is known, composition is also known; likewise, if temperature and pressure in another vessel is known, composition in that vessel is known as well. Although it may not be readily apparent what these compositions are, the point to be made is that they are fixed and can be found to any desired degree of accuracy.

In the method employed here, viscosity, vapor pressure and density are measured in three separate vessels. Fluids for viscosity and vapor pressure are housed in identical $300 \mathrm{ml}$ stainless steel bombs; fluids for density measurements are charged into a glass bulb with a long neck which is equipped with a scribe mark for the purpose of measuring the volume occupied by the known mass of liquid in the bulb. These vessels are depicted conceptually in Figure 1.

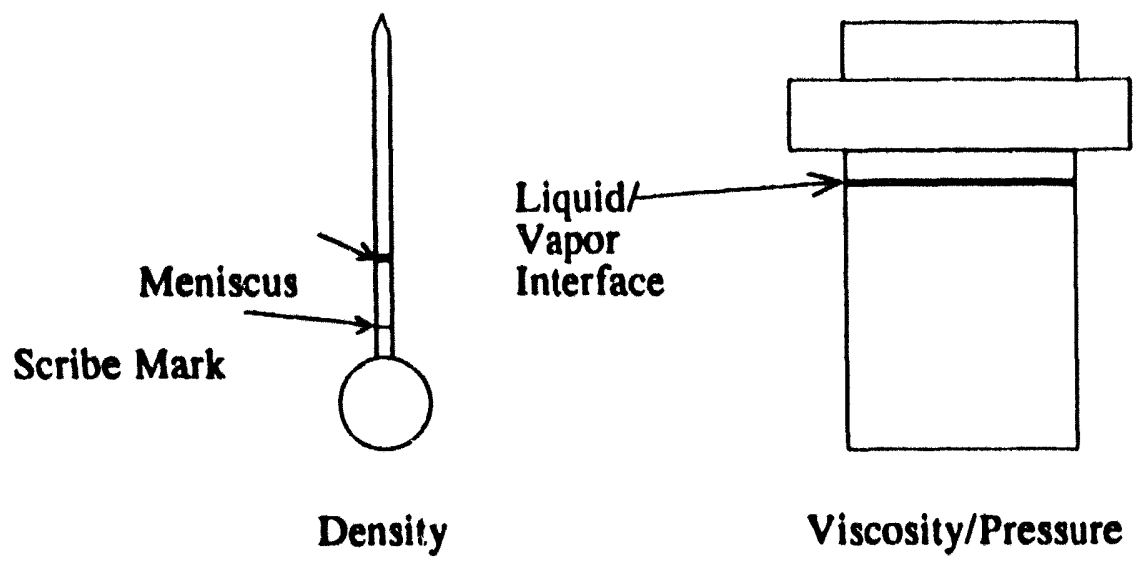

Figure 1: Density Bulb and Viscosity/Pressure Vessels

Density is measured by determining the volume occupied by the liquid as given by readings of meniscus height (w.r.t. scribe mark). Pressure is measured by a variable capacitance transducer, and viscosity is measured by an electromagnetic device; both of these are described in detail in the proposal for this project (and elsewhere), and will not be repeated here.

Since there exists a free volume above the liquid, a portion of the refrigerant charge will occupy this free volume, the amount of which depends on the gas density. The composition of the liquid consequently changes as the temperature is varied by thermally cycling the vessels. Care is taken in charging these vessels to minimize the free volume consistent with safely requirements so that shifts in composition from the "as charged" condition are small.

After charging the vessels, pressure, density and viscosity are experimentally determined as a function of temperature. The "as charged" composition, which is determined to within 0.0015 mass fraction, serves as a boundary condition for an iterative computer program. This program is used to find the composition of the liquid phase over the entire experimental measurement range; the algorithm and program are described below. 


\section{Notatlon:}

$P_{n}(T)=$ pressure as a function of temperature at the "as charged" composition ( $n$ = nominal refrigerant mass fraction, i.e. $0.1,0.2,0.3$ )

$\rho_{n}(T)=$ density as a function of temperature at the "as charged" composition

$\mathrm{P}(\mathrm{T}, \omega)=$ pressure as a function of temperature and composition

$\rho(T, \omega)=$ density

$(\omega=$ refrigerant mass fraction)

$\eta(T, \omega)=$ absolute viscosity

$v(T, \omega)=$ kinematic viscosity

$m_{0}=$ mass of oil

$m_{1}=$ mass of refrigerant

\section{Aleorithm:}

1. Find $P_{n}(T)$; use $P_{n}(T)$ and measured density to calculate composition in density bulbs at each temperature point.

2. Find $\rho_{n}(T)$; use $\rho_{0}(T)$ and measured pressure to calculate composition in pressure bomb at each temperature point.

At this point, data files consisting of the ordered triples temperature, pressure, composition and temperature, density, composition are constructed, where composition is a refined initial guess based on the measured data.

3. Find $P(T, \infty)$.

4. Use $P(T, \omega)$ to refine density bulb compositions.

5. Find $\rho(T, \omega)$.

6. Use $\rho(T, \infty)$ to refine pressure compositions.

7. Repeat steps 3 through 6 until the composition change at any measurement point is less than 0.00001 mass fraction refrigerant.

8. Using final $P(T, \omega)$ and $\rho(T, \omega)$ perform calculations on viscosity vessel until composition change at any measurement point is less than 0.00001 mass

9. Find $\eta(T, \infty)$. fraction refrigerant.

10. Construct a data file containing the ordered triples temperature, kinematic viscosity, composition.

11. Find $v(T, \omega)$.

12. Plot $P(T, \omega)$ and $v(T, \omega)$ for constant $\omega$ on the Daniel Chart.

13. Plot $\rho(T, \omega)$ for constant $\omega$.

\section{Method for calculatine composition:}

1. Use density and mass of liquid to determine volume occupied by liquid phase (using measured density takes volume change on mixing into account).

2. Subtract from total volume to obtain vapor space volume.

3. Use subroutine VIT from REFPROP, with measured temperature and pressure, to find molar volume of refrigerant gas.

4. Subtract mass of refrigerant gas from amount charged to obtain composition. 
Factors which cause shifts in composition to be larger are higher liquid density and higher vapor pressure at lower solution teinperatures. As a specific example, HCFC-22 with ISO 32 naphthenic mineral will be examined in detail.

"As charged" composition of the density bulbs and pressure bombs are given below in Table A-1. Oil and refrigerant amounts are given in grams.

\begin{tabular}{|c|ccc|ccc|} 
& \multicolumn{3}{|c|}{ Densily bulbs } & \multicolumn{3}{c|}{ Pressure bombs } \\
& $\mathbf{m}_{0}$ & $\mathrm{~m}_{1}$ & $\omega$ & $\mathrm{m}_{\mathrm{o}}$ & $\mathrm{m}_{\mathrm{r}}$ & $\omega$ \\
\hline 0.3 & 10.078 & 4.319 & 0.3000 & 141.2 & 60.6 & 0.3003 \\
0.2 & 11.455 & 2.864 & 0.2000 & 160.3 & 40.0 & 0.1997 \\
0.1 & 12.679 & 1.409 & 0.1000 & 180.1 & 20.0 & 0.09995
\end{tabular}

Table A-1: "As Charged" Compositions

Results of the calculations are given in Table A-2, which shows the refined initial estimates, and Table A-3 which gives the compositions after completion of iterations.

\begin{tabular}{|c|c|c|c|c|c|c|}
\hline \multirow{2}{*}{ Temp, C } & \multicolumn{3}{|c|}{ Density Bulb Compositions } & \multicolumn{3}{c|}{ Pressure Bomb Compositions } \\
\cline { 2 - 7 } & $n=0.3$ & $n=0.2$ & $n=0.1$ & $n=0.3$ & $n=0.2$ & $n=0.1$ \\
\hline 0 & 0.2972 & 0.1976 & 0.09902 & 0.2922 & 0.1929 & 0.09581 \\
\hline 20 & 0.2955 & 0.1960 & 0.09849 & 0.2871 & 0.1890 & 0.09359 \\
\hline 40 & 0.2944 & 0.1951 & 0.09840 & 0.2797 & 0.1837 & 0.09085 \\
\hline 60 & 0.2935 & 0.1944 & 0.09868 & 0.2704 & 0.1778 & 0.08755 \\
\hline 80 & 0.2938 & 0.1948 & 0.09935 & 0.2603 & 0.1724 & 0.08498 \\
\hline 100 & 0.2943 & 0.1955 & 0.09985 & 0.2534 & 0.1693 & 0.08393 \\
\hline
\end{tabular}

Table A-2: Density Bulb and Pressure Bomb Compositions After Algorithm Steps 1 and 2

\begin{tabular}{|c|c|c|c|c|c|c|}
\hline \multirow{2}{*}{ Temp, C } & \multicolumn{3}{|c|}{ Density Bulb Compositions } & \multicolumn{3}{c|}{ Pressure Bomb Compositions } \\
\cline { 2 - 7 } & $n=0.3$ & $n=0.2$ & $n=0.1$ & $n=0.3$ & $n=0.2$ & $n=0.1$ \\
\hline 0 & 0.2970 & 0.1974 & 0.09905 & 0.2922 & 0.1929 & 0.09581 \\
\hline 20 & 0.2954 & 0.1958 & 0.09847 & 0.2871 & 0.1890 & 0.09359 \\
\hline 40 & 0.2942 & 0.1948 & 0.09830 & 0.2798 & 0.1838 & 0.09089 \\
\hline 60 & 0.2931 & 0.1938 & 0.09856 & 0.2709 & 0.1780 & 0.08756 \\
\hline 80 & 0.2931 & 0.1941 & 0.09927 & 0.2612 & 0.1727 & 0.08509 \\
\hline 100 & 0.2936 & 0.1956 & 0.09981 & 0.2544 & 0.1697 & 0.08405 \\
\hline
\end{tabular}

Table A-3: Density Bulb and Pressure Bomb Compoittions After Completion of the Iterations 
It can be seen that larger composition shifts are observed in the pressure bombs than in the density bulbs; this is due to the fact that the liquid phase occupies the long, narrow neck of the density bulb, and changes in the height of the liquid phase as the temperature is elevated are large. This causes smaller vapor space volume, which tends to counter the effects of increased pressure; in fact, at the higher temperatures the effect of decreasing vapor space dominates.

Comparison of Tables A-2 and A-3 show that the iteration quickly converges. After the first two steps of the algorithm, the composition is within 0.1 mass fraction of the true composition, and the convergence criteria is satisfied in two iterations of algorithm steps 3 through 7.

The value used for the specific volume of the refrigerant gas in the vapor space is obviously important, and subroutine VIT from REFPROP has been extracted and used for this purpose. The limitations of REFPROP in representing refrigerant properties near the critical point are recognized, and for the "worst case" composition shift in this data set, the value $8.523 \mathrm{cc} / \mathrm{g}$ was obtained for HCFC22 at the measured thermodynamic condition of $100^{\circ} \mathrm{C}$ and $3,113 \mathrm{kPa}$. This compares very favorably with pressure-enthalpy diagrams published by ASHRAE and DuPont.

Having at this point an excellent representation of the pressure-volume-temperature behavior, calculations for the viscosity bomb proceed in a similar iterative fashion, also reaching convergence in two iterations for this fluid. The free volume of the viscosity bomb (as compared to the pressure bomb) is generally lower, which results in smaller composition shifts, as illustrated in Table A.4 (compare with Table A-3).

\begin{tabular}{|c|c|c|c|}
\hline \multirow{2}{*}{ Temp, C } & \multicolumn{3}{|c|}{ Viscosity Bomb Compositions } \\
\cline { 2 - 4 } & $n=0.3$ & $n=0.2$ & $n=0.1$ \\
\hline 0 & 0.2961 & 0.1968 & 0.1040 \\
\hline 20 & 0.2927 & 0.1946 & 0.1031 \\
\hline 40 & 0.2890 & 0.1925 & 0.1022 \\
\hline 60 & 0.2849 & 0.1902 & 0.1012 \\
\hline 80 & 0.2809 & 0.1880 & 0.1004 \\
\hline 100 & 0.2773 & 0.1862 & 0.09968 \\
\hline
\end{tabular}

Table A-4: Vlscosity Bomb Compositions After Completion of the Iterations

Two data files consisting of the ordered triples temperature, absolute viscosity, composition and temperature, kinematic viscosity, composition are now constructed and are used to find these viscosities as a function of temperature and composition. Regression constants and statistical measures of goodness of fit are calculated, and curves of constant composition are plotted from these equations.

Isobaric viscosity curves are generated algebraically, using the assumptions that interpolation between measured curves is valid, the fluid has two degrees of freedom and the vapor pressure of the neat oil is identically zero over the temperature range of interest. These curves are plotted on the upper portion of the Daniel Chart. 


\section{APPENDIX B}

\section{Varlations in Viscosity/Concentration Measurements Among Phase II ARTI Contractors}

During this reporting period, discrepancies were brought to light between the data given in this report, data reported by lowa State University in a previous study, and data taken with a fourth generation apparatus by Imagination Resources, Inc. (IRI). The series of measurcments performed to assist in resolving these discrepancies, along with ARTI communications, is summarized here.

Please provide complete step-by-step details as to how concentration deterninations are made, identify the varlables involved, and provide error-band analysis for each of these variables.

1. Measure the density of the lubricant at room temperature. Using the computer program, determine the mass of refrigerant and lubricant to be charged (this program calculates the amounts to be charged, consistent with minimization of vapor space and safety requirements).

2. Beginning with a scrupulously clean vessel, pour lubricant to the nearest $1.0 \mathrm{gram}$ given by part 1 . Using a disposable syringe, add/remove drops until within $0.1 \mathrm{gram}$. Replace vessel cover and evacuate at $\mathbf{2 0}$ microns for $\mathbf{3 0}$ minutes while stirring magnetically.

3. Cool vessel slightly by immersion in water bath, and condense approximate amount of refrigerant into the vessel. Weigh vessel and repeat until target refrigerant weight is condensed to the nearest $0.1 \mathrm{gram}$ (experience with this procedure allows one to become very efficient).

Possible errors in this procedure are errors in weight only; the balance used determines weight to $+1-0.1$ grams. Table B-1 below shows maximum error for the case under discussion, R-22/Suniso 3GS. It can be seen that excellent precision with small error is obtained with this technique.

\begin{tabular}{|c|c|c|}
\hline $\begin{array}{c}\text { Target Composition } \\
\text { mass fraction }\end{array}$ & $\begin{array}{c}\text { Actual Composition } \\
\text { mass fraction, pressure }\end{array}$ & $\begin{array}{c}\text { Actual Composition } \\
\text { mass fraction, viscosity }\end{array}$ \\
\hline 0.3 & $0.2991+/-0.0012$ & $0.2999+/-0.0014$ \\
0.2 & $0.1997+/-0.0013$ & $0.1991+/-0.0015$ \\
0.1 & $0.0999+/-0.0014$ & $0.1056+/-0.0015$ \\
\hline
\end{tabular}

Table B-1: Pressure and Viscosity Bomb Charges, R-22/Suniso 3GS

Fluids for density determinations are charged in a similar fashion, with the exception that the refrigerant is charged through a calibrated gas manifold and the lubricant is weighed on a more accurate balance due to the smaller amounts of fluids used. The lubricant mass is known to $0.001 \mathrm{gram}$ and the refrigerant mass to 0.004 gram; values for the R-22/Suniso 3GS mixture are given in Table B-2.

\begin{tabular}{|c|c|}
\hline $\begin{array}{c}\text { Target Composition } \\
\text { mass fraction }\end{array}$ & $\begin{array}{c}\text { Actual Composition } \\
\text { mass fraction }\end{array}$ \\
\hline 0.3 & $0.3000+1-0.00032$ \\
0.2 & $0.2000+1-0.00035$ \\
0.1 & $0.1000+1-0.00042$ \\
\hline
\end{tabular}

Table B-2: Density Bulb Charges, R-22/Suniso 3GS 
Please reverify the purity of the Suniso 3GS lubricant that was used in the tests.

Purity of the sample was reverified, with the results given below. Variations from previously reported values are insignificant.

$\begin{array}{ll}\text { Moisture: } & 25 \mathrm{ppm} \\ \text { Total Acid Number: } & 0.02 \mathrm{mg} \mathrm{KOH} / \mathrm{g} \\ \text { Iron Content: } & <1 \mathrm{ppm} \\ \text { Copper Content: } & <1 \mathrm{ppm}\end{array}$

Please provide viscosity measurements of the pure Suniso 3GS lubricant (e.g., without refrigerant) - over a range of 0 to $100^{\circ} \mathrm{C}$ - that were obtained utilizing your individual procedures for determining viscosities of lubricant-refrigerant mixtures.

The dynamic viscosity measurements taken for pure Suniso 3GS are given in table B-3. In order to provide kinematic viscosity data for comparison, it is necessary to divide the dynamic viscosity by density; since the temperatures at which viscosity measurements were taken do not exactly coincide with the temperatures at which density measurements were obtained, the best fit linear equation was used to calculate density at each temperature for which a viscosity measurement exists. Table B-4 includes the density points and the equation; Table B-5 gives the kinematic viscosity data.

\begin{tabular}{|c|c|c|c|c|c|}
\hline T, C & Viecoaity & $\mathbf{T}, \mathbf{C}$ & Vlecosily & $T, C$ & Viecosily \\
\hline T.3T & क्.1. & 34.44 & 35.3 & 67.47 & 8.93 \\
\hline 2.55 & 404.8 & 35.48 & 33.6 & 68.48 & 8.65 \\
\hline 3.76 & 356.6 & 36.46 & 31.9 & 69.36 & 8.15 \\
\hline 2.37 & 323.3 & 37.31 & 30.2 & 70.56 & 8.17 \\
\hline 5.54 & 299.1 & 38.52 & 28.6 & 71.31 & 7.91 \\
\hline 6.68 & 263.1 & 39.46 & 27.2 & 72.67 & 7.37 \\
\hline 7.75 & 240.8 & 40.50 & 25.8 & 73.64 & 7.40 \\
\hline 8.62 & 220.8 & 11.30 & 24.3 & 74.48 & 7.16 \\
\hline 9.37 & 203.4 & 21.35 & 23.4 & 75.36 & 7.00 \\
\hline 10.44 & 188.8 & 73.54 & 22.3 & 76.42 & 6.83 \\
\hline 11.30 & 175.7 & 4.69 & 21.1 & 77.54 & 6.33 \\
\hline 12.47 & 160.2 & 43.48 & 20.3 & 78.52 & 6.41 \\
\hline 13.63 & 14.0 & 46.50 & 19.4 & 79.31 & 6.22 \\
\hline 14.45 & 133.1 & 77.34 & is.5 & 80.34 & $6 . \overline{06}$ \\
\hline 13.4 & 125.1 & 48.34 & 17.7 & 81.37 & 3.96 \\
\hline 16.46 & 113.3 & $\$ 9.16$ & 17.1 & 82.44 & 5.86 \\
\hline 17.37 & 105.6 & 50.5I & 16.4 & 83.54 & 3.71 \\
\hline 18.33 & 98.4 & 31.31 & 15.5 & 84.44 & 3.49 \\
\hline 19.46 & बा.5 & 32.54 & TS.T & 85.38 & 3.29 \\
\hline 20.43 & 85.6 & 33.46 & 14.7 & 86.30 & 9.32 \\
\hline 21.49 & 79.3 & 34.47 & 14.1 & 87.49 & 5.11 \\
\hline 22.35 & 73.8 & 55.50 & 13.3 & 78.66 & 7.97 \\
\hline 23.70 & 89.1 & 36.50 & 13.0 & 89.42 & 9.86 \\
\hline 24.49 & 64.5 & 37.49 & 12.5 & 90.65 & 9.73 \\
\hline 25.57 & 60.2 & 38.53 & 12.1 & 91.63 & 4.60 \\
\hline 26.55 & 56.5 & 39.49 & 11.7 & 92.60 & 4.98 \\
\hline 27.32 & 32.9 & 60.42 & 11.3 & 93.35 & 7.41 \\
\hline 28.35 & 49.7 & 01.47 & 10.8 & 94.30 & 7.39 \\
\hline 29.35 & $\$ 6.6$ & $\mathbf{6 2 . 3 2}$ & 10.5 & क्व.41 & 7.27 \\
\hline $30 . \overline{32}$ & 73.9 & 63.33 & 10.1 & 96.62 & 4.16 \\
\hline 31.32 & वा. & 64.47 & 9.60 & 97.80 & 4.17 \\
\hline 32.79 & 39.2 & 85.36 & 9.19 & 98.35 & 4.17 \\
\hline 33.46 & 37.1 & 66.36 & 9.16 & 99.62 & 7.04 \\
\hline
\end{tabular}

Table B-3: Dynamic Viscosity

\begin{tabular}{|c|c|c|c|c|c|}
\hline $\mathbf{T}, \mathbf{C}$ & $\begin{array}{c}\text { Viscosity } \\
\text { cat }\end{array}$ & $\mathbf{T}, \mathbf{C}$ & $\begin{array}{c}\text { Viecosity } \\
\text { cat }\end{array}$ & $\mathbf{T}, \mathbf{C}$ & Visconity \\
\hline 1.51 & 481.0 & 34.44 & 39.4 & 67.47 & 10.2 \\
\hline 2.35 & 441.8 & 35.48 & $3 \overline{7} .5$ & 68.48 & $\overline{9.89}$ \\
\hline 3.76 & 389.5 & 36.78 & 33.6 & 69.36 & 9.63 \\
\hline 4.37 & 335.4 & 37.31 & 33.8 & 70.36 & 9.33 \\
\hline 3.34 & 327.2 & 38.52 & 32.1 & 71.31 & $9 . \overline{06}$ \\
\hline 6.68 & 288.0 & 39.46 & 30.5 & $\overline{72.67}$ & $8 . \overline{68}$ \\
\hline 7.75 & 263.7 & 40.50 & 29.0 & 73.64 & 8.48 \\
\hline 8.62 & 242.0 & 41.30 & 27.6 & 74.48 & 8.22 \\
\hline 9.37 & -223.2 & $\mathbf{4 2 . 3 5}$ & 26.5 & 73.36 & 8.04 \\
\hline 10.44 & 207.2 & 43.54 & 25.1 & 76.42 & 7.85 \\
\hline 11.30 & 192.9 & 44.69 & 23.7 & $\overline{77.54}$ & 7.52 \\
\hline 12.47 & 176.1 & $\mathbf{4 5 . 4 8}$ & 22.9 & $\overline{78.52}$ & 7.38 \\
\hline 13.63 & 138.4 & 76.30 & 21.9 & 79.31 & 7.16 \\
\hline 14.43 & 748.6 & 47.34 & 20.9 & 80.34 & 6.99 \\
\hline 15.44 & 137.7 & 48.54 & 20.0 & 81.37 & 6.88 \\
\hline 16.46 & 127.0 & 49.46 & 19.3 & 82.44 & 6.77 \\
\hline 17.57 & 116.5 & 50.51 & 18.6 & 83.54 & $6 . \overline{60}$ \\
\hline 18.35 & 108.6 & 31.34 & 17.9 & 84.44 & 6.36 \\
\hline 19.48 & 101.0 & 32.34 & 17.1 & 83.38 & 6.12 \\
\hline 20.43 & 94.6 & 33.46 & 16.6 & 86.30 & $6.1 \overline{6}$ \\
\hline 21.49 & 87.6 & 54.47 & 16.0 & $87 . \overline{49}$ & $\overline{3} . \overline{92}$ \\
\hline 22.32 & 81.6 & 35.30 & 15.3 & 88.68 & 3.77 \\
\hline 23.48 & 76.5 & 56.30 & 14.8 & 89.42 & 3.64 \\
\hline 24.49 & $\pi .3$ & 37.79 & 14.2 & 90.63 & 5.63 \\
\hline 25.37 & 66.7 & 58.53 & 13.7 & 91.63 & 5.36 \\
\hline 26.55 & 62.7 & 59.49 & 1.33 & $92 . \overline{60}$ & $-\overline{5} . \overline{35}$ \\
\hline 27.32 & 38.8 & 60.12 & 12.8 & 93.35 & 3.18 \\
\hline 28.35 & 35.3 & 61.47 & 12.3 & 94.30 & 3.12 \\
\hline 29.35 & 31.9 & 62.32 & 11.9 & 95.41 & 7.98 \\
\hline 30.32 & 48.9 & 63.53 & II.J & $96 . \overline{62}$ & $4 . \overline{86}$ \\
\hline $31 . \overline{52}$ & 46.1 & 64.47 & 11.3 & 97.80 & 4.87 \\
\hline 32.49 & -43.7 & 63.36 & 10.8 & 98.33 & 7.87 \\
\hline 33.46 & 91.4 & 66.36 & 10.5 & 99.62 & $4 . \overline{72}$ \\
\hline
\end{tabular}

Table B-5: Kinematic Viscosity

\begin{tabular}{|c|c|c|c|c|c|c|c|c|c|c|c|}
\hline$\overline{\mathbf{T}}, \overline{\mathbf{C}}$ & 0.5 & 10.8 & 18.9 & 24.4 & $30 . \overline{0}$ & 40.0 & 50.0 & 60.0 & $\overline{70} . \overline{0}$ & 80.0 & $\overline{9} \overline{0} . \overline{0}$ \\
\hline $\begin{array}{l}\text { gensiny } \\
\text { ece }\end{array}$ & 0.9174 & 0.9105 & 0.9048 & 0.9015 & 0.8978 & 0.8917 & 0.8851 & 0.8973 & 0.8728 & 0.8663 & 0.8598 \\
\hline
\end{tabular}

Table B-4: Density 
Most importantly, individual attention to the following identified items are also required: although it was acknowledged that your method should result in accurate results, especially if the vapor head space is $\mathrm{m} / \mathrm{n} / \mathrm{mized}$, it was noted that physical measurements are hard to beat. Accordingly, please determine viscosity and refrigerant concentration values for a $R$ $22 /$ mineral oll mixture at $40^{\circ} \mathrm{C}$ and $100^{\circ} \mathrm{C}$ (near suggested pressures noted below) using your standard procedure and by taking physical weight measurements of the refrigerant-rich lubricant. It was opined that weighing physical samples should be relatively easy to accomplish if you can Invert the flask (1.e., use the vapor port as a liquid port) or add an extra tap.

In order to address this question, we have completed the full treatment of the data set as given in our proposal and as detailed in this report. The viscosity data is combined into one master file consisting of temperature, viscosity, composition ordered triples, as is the pressure data and the density data, and least squares regression is performed on these files in order to find viscosity, pressure and density as a function of the variables temperature and composition.

These equations have been used to interpolate between measured curves so that data may be provided at the requested temperatures and pressures, which are given in Tables B-6 and B-7 below. The entries " $W>0.3^{\prime \prime}$ and " $>0.3^{n}$ mean that the refrigerant mass fraction at the temperature and pressure indicated is greater than 0.3 , which is outside the measured range.

\begin{tabular}{|c|c|c|}
\hline Pressure, psia & Viscosity, cst at 40 C & Viscosity, cst at 100 C \\
\hline 50 & 16.3 & 3.16 \\
\hline 75 & 10.6 & 2.97 \\
\hline 100 & 7.21 & 2.79 \\
\hline 150 & 3.85 & 2.47 \\
\hline 200 & $w>0.3$ & 2.19 \\
\hline 225 & $w>0.3$ & 2.08 \\
\hline 325 & $w>0.3$ & 1.68 \\
\hline 400 & $w>0.3$ & 1.46 \\
\hline
\end{tabular}

Table B-6: Kinematic Viscosity of HCFC-22/Suniso 3GS at Selected Temperatures and Pressures

\begin{tabular}{|c|c|c|}
\hline Pressure, psia & $\begin{array}{c}\text { Refrigerant Mass Fraction at } \\
40 \mathrm{C}\end{array}$ & $\begin{array}{c}\text { Refrigerant Mass Fraction at } \\
100 \mathrm{C}\end{array}$ \\
\hline 50 & 0.036 & 0.014 \\
\hline 75 & 0.085 & 0.029 \\
\hline 100 & 0.134 & 0.044 \\
\hline 150 & 0.231 & 0.075 \\
\hline 200 & $>0.3$ & 0.106 \\
\hline 225 & $>0.3$ & 0.121 \\
\hline 325 & $>0.3$ & 0.183 \\
\hline 400 & $>0.3$ & 0.229 \\
\hline
\end{tabular}

Table B-7: Refrigerant Concentrations of HCFC-22/Suniso 3GS at Selected Temperatures and Pressures 


\section{Subsequent ARTI Requests and Measurements}

Following the above measurements, it was requested that SAI verify, with experimental data, the calculations performed to correct for the vapor space volume. To accomplish this, a 300 $\mathrm{ml}$ bomb identical to the ones used to measure viscosity and pressure was charged with Suniso 3GS and R-22, at various concentrations, and allowed to reach thermal equilibrium at room temperature, 40 and $100^{\circ} \mathrm{C}$. A sample (about $80 \mathrm{grams}$ ) was then withdrawn from the liquid phase of the bomb, and the amounts of refrigerant and lubricant in this sample were determined by weight measurements. In order to remove the R-22 from the oil, the sample was heated for four hours at $100^{\circ} \mathrm{C}$ and then vacuumed at 20 microns for 30 minutes.

The calculation procedure employs REFPROP to give the spexific volume of the refrigerant gas at the measured conditions. As mentioned above, the limitations of this program in representing refrigerant properties near the critical point are recognized; for the conditions at hand, the specific volumes calculated by REFPROP have been checked against publications by ASHRAE and DuPont, and have been found to agree quite well with these sources.

Table B-8 gives the temperature and pressure of the solutions in columns 1 and 2 . Column 3, "Composition as Charged," simply reflects the weights of refrigerant and lubricant introduced. Column 4, "Corrected Composition," is the composition as calculated by the correction procedure - these are the values used in the numerical analysis reported here. The fifth column, "Measured Composition," is the composition of the sample as determined by weight measurements.

\begin{tabular}{|c|c|c|c|c|}
\hline Temp, C & Pressure, psia & $\begin{array}{c}\text { Composition as } \\
\text { Charged }\end{array}$ & $\begin{array}{c}\text { Corrected } \\
\text { Composition }\end{array}$ & $\begin{array}{c}\text { Measured } \\
\text { Composition }\end{array}$ \\
\hline 23.9 & 115.0 & 0.2754 & 0.2680 & 0.2668 \\
\hline 40 & 47.8 & 0.0362 & 0.0320 & 0.0309 \\
\hline 40 & 141.3 & 0.2307 & 0.2140 & 0.2117 \\
\hline 100 & 46.0 & 0.0141 & 0.0112 & 0.0088 \\
\hline 100 & 164.3 & 0.1059 & 0.0839 & 0.0849 \\
\hline 100 & 326.9 & 0.2286 & 0.1838 & 0.1826 \\
\hline
\end{tabular}

Table B-8: Compositions in Refrigerant Mass Fraction at Selected Temperatures and Pressures

Comparison of column 3 with column 5 reveals that significant differences exist between the composition as charged and the true composition; these differences become more pronounced as the temperature and pressure are elevated.

Comparison of the fourth and fifth columns will show that the calculation procedure is accurate to within 0.0024 mass fraction (or 0.24 mass percent). If, assuming the worst case, this is additive to the uncertainty in weight measurements and the fifth column above is exactly correct, the total uncertainty in composition is $+1-0.004$ mass fraction (or 0.4 weight percent).

Following this exercise, further measurements were requested by $\mathrm{ARTI}$, which SAI declined to perform for the following reasons: (1) it was requested that an experimental technique be implemented which has been investigated by us in the past with poor results, (2) the calculation procedures which correct for composition shifts were adequately verified by the above and (3) further measurements, as was the work reported in this Appendix, is beyond the scope of the contract and would not in our opinion bring this issue closer to a resolution. 
At this time, in order to take a fresh look, Table B-9 was prepared, which compares pressure versus temperature and composition data reported by SAI and Iowa State University (ISU) ${ }^{1}$.

\begin{tabular}{|c|c|c|c|c|c|c|}
\hline \multirow{2}{*}{$\begin{array}{c}\text { Temp, } \\
\text { Celsius }\end{array}$} & \multicolumn{4}{|c|}{ SAI } & \multicolumn{4}{c|}{ ISU } \\
\cline { 2 - 7 } & .1 & .2 & .3 & .1 & .2 & .3 \\
\hline 0 & 25.9 & 42.9 & 59.8 & -- & -- & -- \\
\hline 20 & 52.8 & 83.0 & 113.1 & -- & -- & -- \\
\hline 40 & 82.7 & 134.1 & 185.4 & 86.1 & 147.7 & 183.7 \\
\hline 60 & 115.7 & 196.2 & 276.7 & 123.3 & 209.7 & 259.6 \\
\hline 80 & 151.6 & 269.2 & 386.9 & 163.1 & 284.4 & 363.9 \\
\hline 100 & 190.6 & 353.3 & 516.1 & 206.2 & 371.8 & 496.9 \\
\hline
\end{tabular}

Table B-9: Pressure (psia) at Selected Temperatures and Compositions

It can be seen from the above that the measurements agree within $5 \%$ on the average; these data also compare favorably with data published by Witco Chemical $\mathrm{Co}^{2}$. After review of the supporting data submitted by both laboratories (SAI and IRI), the belief was expressed by ARTI that the differences were attributable to the error bands of the two vastly different measuring techniques; it was also recognized that measurement of refrigerant concentration values near the critical point is a difficult task.

'Van Gaalen, N. A., Zoz, S. C. and Pate, M. B., "The Solubility and Viscosity of Solutions of HCFC-22 in Naphthenic Oil and in Alkylbenzene at High Pressures and Temperatures," ASHRAE Transactions 1991, Vol. 97, Pt. 1.

${ }^{2}$ Witco Chemical Co., Sonneborn Division, "Witco SUNISO Refrigeration Oils," Technical Bulletin Number 8846. 


\section{APPENDIX C}

\section{Lubricant Purity}

Moisture, total acid number, iron content and copper content have been measured for the lubricants reported in this study. Table C-1 below gives these results. The intent of these measurements is to verify purity of the lubricants prior to study of the viscosity, solubility and density characteristics when mixed with various refrigerants; impurities of the order shown below have negligible effects on these properties. Lubricants were stored in the container as shipped from the manufacturer untile used, at which time the values were measured. Storage times ranged from zero to four months.

\begin{tabular}{|c|c|c|c|c|}
\hline LUBRICANT & Moisture, ppm & $\begin{array}{c}\text { Total Acid Number, mg } \\
\text { KOH/g oil }\end{array}$ & Fe, ppm & Cu, ppm \\
\hline $\begin{array}{c}\text { ISO 32 Naphthenic } \\
\text { Mineral Oil }\end{array}$ & 31 & 0.02 & $<1$ & $<1$ \\
\hline $\begin{array}{c}\text { ISO 100 Naphthenic } \\
\text { Mineral Oil }\end{array}$ & 42 & 0.03 & $<1$ & $<1$ \\
\hline $\begin{array}{c}\text { ISO 22 Pentaerythritol } \\
\text { Ester Mixed Acid }\end{array}$ & 35 & $<0.01$ & $<1$ & $<1$ \\
\hline $\begin{array}{c}\text { ISO 32 Pentaerythritol } \\
\text { Ester Mixed Acid } \| 1\end{array}$ & 35 & $<0.01$ & $<1$ & $<1$ \\
\hline $\begin{array}{c}\text { ISO 68 Pentaerythritol } \\
\text { Ester Mixed Acid }\end{array}$ & 77 & 0.04 & $<1$ & $<1$ \\
\hline $\begin{array}{c}\text { ISO 100 Pentaerythritol } \\
\text { Ester Mixed Acid }\end{array}$ & 80 & 0.06 & $<1$ & $<1$ \\
\hline $\begin{array}{c}\text { ISO 32 Pentaerythritol } \\
\text { Ester Mixed Acid } \| 2\end{array}$ & 64 & 0.04 & 4 & $<1$ \\
\hline 150 SUS Alkylbenzene & 29 & 0.01 & 0.04 & $<1$ \\
\hline 300 sUS Alkylbenzene & 29 & & $<$ \\
\hline
\end{tabular}

Table C-1: Lubricant Purity 


\section{APPENDIX D}

\section{Commercial Identification}

Lubricants tested are commercially available and are:

ISO 32 naphthenic mineral oil

ISO 100 naphthenic mineral oil

ISO 22 pentaerythritol ester mixed acid

ISO 32 pentaerythritol ester mixed acid $\# 1$

ISO 68 pentaerythritol ester mixed acid

ISO 100 pentaerythritol ester mixed acid

ISO 32 pentaerythritol ester mixed acid \#2 150 SUS alkylbenzene

300 SUS alkylbenzene
Witco Suniso 3GS

Witco Suniso 5GS

Mobil Arctic EAL 22

Mobil Arctic EAL 32

Castrol Icematic SW 68

Castrol Icematic SW 100

Castrol Icematic SW 32

Shrieve Chemical Zerol 150

Shrieve Chemical Zerol 300 


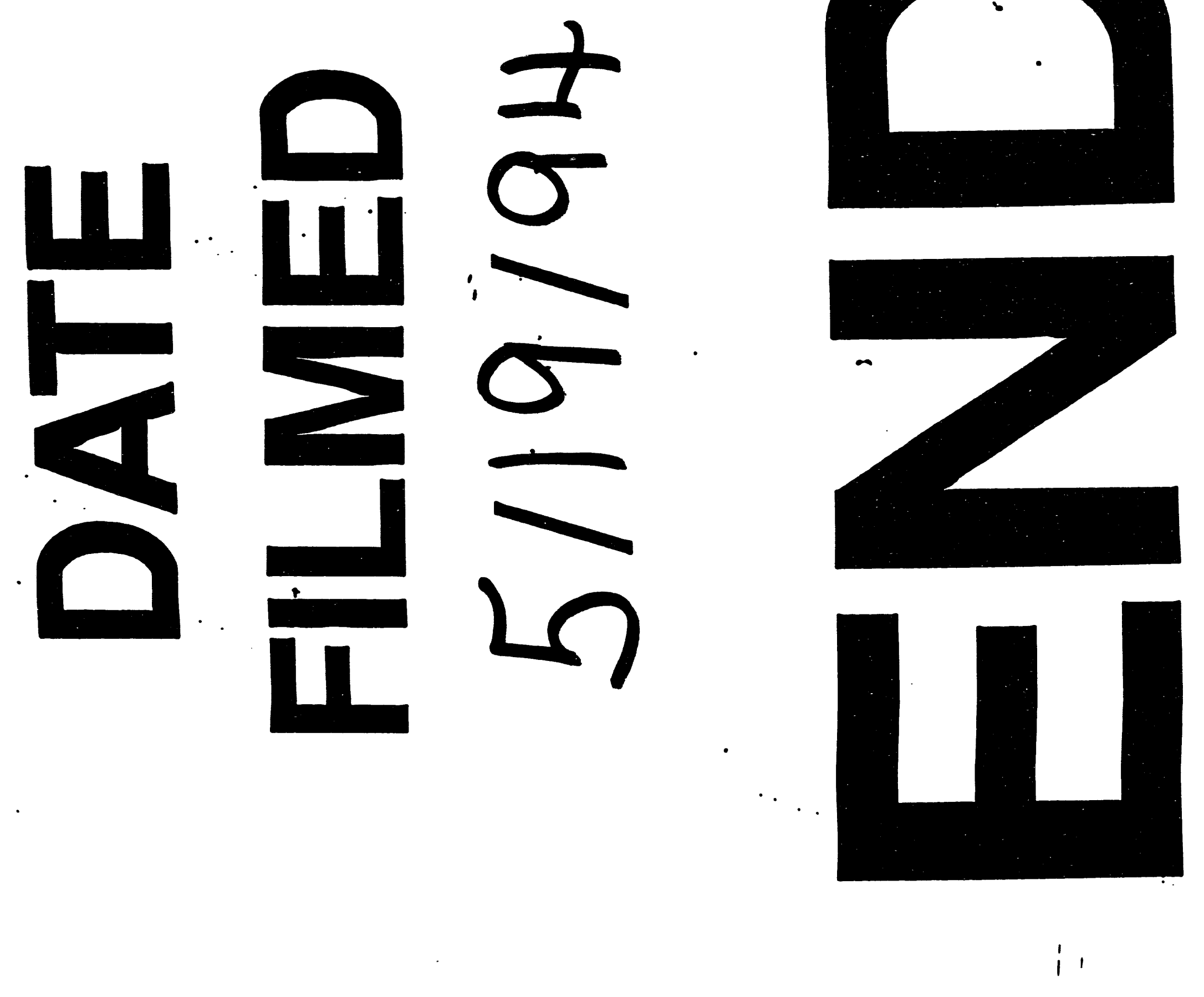


